\title{
Factor investing: alpha concentration versus diversification
}

\author{
Lars Heinrich $^{1} \cdot$ Antoniya Shivarova ${ }^{2} \cdot$ Martin Zurek $^{2}$ (])
}

Revised: 16 May 2021 / Accepted: 17 May 2021 / Published online: 2 June 2021

(c) The Author(s) 2021

\begin{abstract}
Despite extensive research support, the role of diversification in current factor investing strategies remains neglected. This paper investigates whether well-designed multifactor portfolios should not only be based on firm characteristics, but should also include portfolio diversification effects. While the alpha concentration approach mainly considers factor-specific firm characteristics, the diversified approach utilizes covariance estimators in addition to firm characteristics to account for portfolio diversification. The corresponding out-of-sample results show that including an efficient covariance estimator improves the performance of long-only multifactor portfolios compared to the pure alpha concentration approach. A particular advantage of diversified factor investing strategies can be identified in the significant increase in exposure to the low-volatility factor represented by firm characteristics with high informational content. No significant performance differences are observed for long-short portfolios where the factor exposures of the alpha concentration and diversification approaches are similar with respect to the low-volatility factor.
\end{abstract}

Keywords Factor investing · Alpha forecasting · Diversification · Optimal orthogonal portfolio $\cdot$ Information coefficient · Covariance

JEL Classification $\mathrm{G} 11 \cdot \mathrm{G} 12 \cdot \mathrm{G} 15 \cdot \mathrm{G} 17$

Factor investing, also labeled smart beta, has recently become a well-known alternative to market capitalized index investing. Apart from market risk, factor investing tilts portfolio weights towards other specific risk factors, such as value or momentum; and aims to outperform the corresponding capitalization-weighted benchmark.

In practice, factor exposures are realized with simple scoring approaches based on predefined firm characteristics. The cost-effectiveness of these rule-based methodologies is one of the main advantages of factor investing

Martin Zurek

zurek@europa-uni.de

Lars Heinrich

lars.heinrich@wwasset.de

Antoniya Shivarova

shivarova@europa-uni.de

1 Department of Liquid Assets, W\&W Asset Management GmbH, Tambourstraße 1, 70806 Kornwestheim, Germany

2 Department of Finance and Capital Market Theory, European University Viadrina, Große Scharrnstraße 59, 15230 Frankfurt (Oder), Germany strategies. Nevertheless, an overly simplistic adaptation of factor investing strategies may lead to many misunderstandings. Amenc et al. (2016a) discuss ten misconceptions and separate these into groups, such as performance drivers, investability hurdles and strategy choices. ${ }^{1}$ One of the strategy choice misconceptions is represented by the concentration fallacy, stating that a good factor index should provide a strong tilt to the desired factor. Although diversification is an essential cornerstone of modern portfolio theory, this misconception illustrates that implementing diversified factor investing strategies is still a challenging task.

The benefits of diversified factor investing strategies have been proposed by Amenc et al. (2016b) in a single factor environment and by Amenc et al. (2017) for multifactor strategies. The research results imply higher performance outcomes for diversified factor portfolios than for concentrated factor portfolios. Within the security selection approaches of the multifactor strategies, the authors used a simplified factor construction method with equally weighted firm characteristics. Since equal treatment of firm characteristics neglects potential differences in their informational 1 Arnott et al. (2019) stress further problems such as overfitting, crowding and nonnormal drawdowns. 
content, the proposed procedure can lead to biased results. In particular, Heinrich and Zurek (2019) have shown, that naive stock selection models can be significantly improved by considering differences in the informational content between firm characteristics. Since a poorly calibrated stock selection model can reduce the performance of pure firm characteristic-based strategies, our study analyzes the benefits of diversified versus concentrated multifactor portfolios taking into account the full range of interaction effects between firm characteristics.

We present factor investing strategies from the perspective of the optimal orthogonal portfolio (OOP) formally introduced by MacKinlay and Pástor (2000). From the OOP perspective, it is possible to resemble the practical view of an investor who aims to beat a given benchmark portfolio in terms of mean-variance efficiency. Moreover, the OOP perspective provides a motivation for both strategies: A pure firm characteristic-based approach and a diversified strategy that additionally considers the covariance structure of the returns of the selected securities. More specifically, MacKinlay and Pástor (2000) use a strong assumption ${ }^{2}$ regarding the covariance structure of the residual returns to show that the OOP can be weighted proportional to the securities' alphas without explicit consideration of the covariance matrix. As firm characteristics can be transformed into alphas, the OOP perspective motivates purely characteristic-based factor strategies.

In the diversified strategy, we weaken the strong assumption regarding the covariance structure and account for diversification effects by considering a nonzero covariance structure of the residual returns. Moreover, we keep our analysis contemporary and use two efficient state-of-the-art covariance estimation methods. Both estimators are more stable than a sample estimator, especially in a high-dimensional scenario. Due to its popularity among practitioners, the well-known linear shrinkage estimator of Ledoit and Wolf (2004) with a constant correlation structure of returns is adopted as the first estimator. The second estimator follows Amenc et al. (2012) as well as Amenc et al. (2011) and incorporates a factor-based approach to covariance estimation using principal component analysis (PCA) to determine the underlying risk factors. This method is chosen to keep the results comparable to the literature on factor strategies considering diversification effects.

To study the benefits of diversified versus concentrated strategies, we apply a horse race between a pure alpha-based weighting method and a diversification-based strategy. Both strategies are compared considering a comprehensive

\footnotetext{
2 The covariance of the residual returns from a factor model without any omitted risk factor is assumed to be diagonal and proportional to the identity matrix.
}

multifactor model. A multifactor perspective is especially important for practice since in multifactor portfolios and even in single-factor portfolios, many investors try to diversify their factor investing risk by examining several firm characteristics. With regard to the practical applicability of our study, the approaches are analyzed with factors defined according to an industry standard of the MSCI factor portfolios. Overall, the entire multifactor portfolio contains 16 firm characteristics. Moreover, to validate the robustness of the results, two different datasets consisting of stocks from the S\&P 500 and from STOXX Europe 600 are included.

Our empirical findings show that applying the residual covariance matrix improves the out-of-sample performance in long-only multifactor strategies. Diversification strategies manage to both increase excess returns and reduce portfolio risk. By overweighting low-volatility stocks, the low-volatility factor exposure is strongly increased compared to other factors with less relevance. Since the low-volatility factor comprises of firm characteristics with a significant positive informational content, the increase in low-volatility factor exposure provides an important explanatory perspective that has not been recognized in previous research.

In the long-short portfolios, the low-volatility factor exposure in the alpha concentration approach is similar to that in the diversification approaches. As a result, no significant difference in performance is observed. Hence, the informational content structure of the applied firm characteristics and, in particular, the informational content of the low-volatility firm characteristics, can be identified as an important performance driver. Since our alpha forecasting model considers the differences in informational content between the firm characteristics, it allows for an appropriate comparison between the two strategies.

The remainder of the paper is organized as follows. Section 1 introduces the OOP perspective and the factor investing strategies. Section 2 presents the data set and the applied firm characteristics, whereas Sect. 3 reports the findings of the empirical backtest. This includes the results of the longonly and long-short portfolios. Finally, Sect. 4 contains some concluding remarks.

\section{Factor investing strategies}

In practice, factor investing addresses the case of an investor who aims to beat a given benchmark. Therefore, we follow Treynor and Black (1973) by separating the investment universe into an active portfolio $\mathbb{A}$ with $i=1, \ldots, N$ securities and a passive benchmark portfolio $\mathbb{B}$.

The main goal of our analysis is to compare the performance of a pure characteristic-based weighting approach with a scenario that considers diversification effects. Thus, 
we focus on the investment decision ${ }^{3}$ for $A$ and assume the following linear relation between the securities' returns in $\mathbb{A}$ and $\mathbb{B}$ :

$$
\begin{aligned}
R_{\AA}^{*}, t & =\alpha+\beta R_{\mathbb{B}, t}^{*}+\epsilon_{t} \\
\mathrm{E}\left[\epsilon_{t}\right] & =0 \\
\mathrm{E}\left[\epsilon_{t} \epsilon_{t}^{\prime}\right] & =\Sigma
\end{aligned}
$$

$\operatorname{Cov}\left[R_{\mathbb{B}, t}^{*}, \epsilon_{t}\right]=0$,

where the $(N \times 1)$-vector $R_{\mathrm{A}, t}^{*}$ denotes the $N$ security returns in $\mathbb{A}$ at time $t$, and $R_{\mathbb{B}, t}^{*}$ denotes the return on the benchmark portfolio. Throughout the article, the term "return" on a given security denotes the return in excess of the risk-less rate. ${ }^{4}$ The $(N \times 1)$-vector $\beta$ represents the risk sensitivities of the securities returns towards the benchmark returns and the $(N \times 1)$-vector $\epsilon_{t}$ represents the resulting residuals assumed to have zero-mean and a nonzero covariance, denoted as the $(N \times N)$-matrix $\Sigma$.

Since MacKinlay and Pástor (2000) define the $(N \times 1$ )-vector $\alpha$ as the vector of mispricings, we refer to this model as an incomplete factor model. Investors implementing factor strategies believe that factor model (1) can be complemented by an additional factor portfolio $\mathrm{A}$, so that mispricing disappears with $\alpha=\mathbf{0}$. Accordingly, the complemented model is then defined as:

$$
\begin{aligned}
R_{\AA, t}^{*} & =\beta R_{\mathbb{B}, t}^{*}+\beta_{\AA} R_{\AA}^{*}+v_{t} \\
\mathrm{E}\left[v_{t}\right] & =\mathbf{0} \\
\mathrm{E}\left[v_{t} v_{t}^{\prime}\right] & =\Omega
\end{aligned}
$$$$
\operatorname{Cov}\left[R_{\mathbb{B}, t}^{*}, v_{t}\right]=\mathbf{0}
$$$$
\operatorname{Cov}\left[R_{\mathrm{A}, t}^{*}, v_{t}\right]=\mathbf{0} \text {. }
$$

Assuming that the returns of $\mathbb{A}$ and $\mathbb{B}$ are uncorrelated, $\mathbb{A}$ represents the OOP, which corresponds to the missing riskfactor portfolio. The weights for the optimal orthogonal portfolio $a_{\AA}$ can be determined by:

$a_{\AA}=\frac{\Sigma^{-1} \alpha}{\mathbf{1}_{N}^{\prime} \Sigma^{-1} \alpha}$.

In accordance with Fama-French factors, the securities of $\mathbb{A}$ are separated into long and short portfolios. Stocks with alphas above (below) a specified quantile $\alpha_{q t}^{+}\left(\alpha_{q t}^{-}\right)$are assigned to the long (short) portfolio. Since $\alpha_{q t}^{+}$and $\alpha_{q t}^{-}$determine the number of selected securities, these are referred to as selection thresholds. Formally this division is expressed by

\footnotetext{
${ }^{3}$ The performance objective of portfolio $\mathbb{A}$ is to optimize the information ratio. A possible next step would be to optimize the Sharpe ratio of the overall portfolio by combining both portfolios, $A$ and $\mathbb{B}$.

${ }^{4}$ Excess returns are marked with an asterisk.
}

$a_{\mathrm{A}, \text { long }}=\frac{\Sigma_{+}^{-1} \alpha^{+}}{1^{\prime} \Sigma_{+}^{-1} \alpha^{+}} \quad$ and $\quad a_{\mathrm{A}, \text { short }}=\frac{\Sigma_{-}^{-1} \alpha^{-}}{1^{\prime} \Sigma_{-}^{-1} \alpha^{-}}$.

In $a_{\mathrm{A}, \text { long }}$, the alpha vector $\alpha^{+}$and the covariance matrix $\Sigma_{+}$hold the values for the respective securities in the long portfolio. Equivalently, $\alpha^{-}$and $\Sigma_{-}$hold the values for the securities in the short portfolio $a_{\mathrm{A} \text {, short }}$. In addition to longshort (LS) portfolios, we construct long-only (LO) portfolios that consider only the long portfolio. Within the diversification approach, we further need to include no-short-sale constraints to ensure the separation of securities into long and short portfolios. The weighting strategy for $A$ can be applied to build bottom-up multifactor portfolios. The OOP perspective has been applied by Zurek and Heinrich (2021) to show the benefits of bottom-up versus top-down factor strategies. According to this approach, we can apply multiple factor-specific firm characteristics and build multifactor portfolios within a one-step approach.

To obtain the alpha forecasts $\hat{\alpha}$, we use the same linear model as described by Heinrich and Zurek (2019). In this model, the assumption is made that the security returns in $\mathbb{A}$ follow a multivariate conditional normal distribution. The $M$ different types of firm characteristics, hereinafter referred to as signals, can be observed for each of the $N$ companies and represent the necessary conditioning information for each security. For simplification purposes, it is assumed that these signals only have informational value for their specific company, which means that signals from different companies do not correlate with each other or with returns from other companies. Further, we assume that signals are uncorrelated with benchmark returns, so that benchmark timing can be disregarded. Under these conditions, it is possible to apply alpha forecasts with the following linear model

$\hat{\alpha}_{i, t}=\hat{k} \widehat{C}^{-1} z_{i, t-1}$,

with

$$
\begin{aligned}
\hat{k} & =\left(\widehat{\operatorname{Corr}}\left[R_{t}^{*}, g_{1, t-1}\right] \cdots \widehat{\operatorname{Corr}}\left[R_{t}^{*}, g_{M, t-1}\right]\right), \\
z_{i, t-1} & =\left(\begin{array}{c}
\frac{\left(g_{i, 1, t-1}-\widehat{\mathrm{E}}\left[g_{1, t-1}\right]\right)}{\widehat{\sigma}_{g_{1, t-1}}} \\
\vdots \\
\frac{\left(g_{i, M, t-1-1}-\widehat{\mathrm{E}}\left[g_{M, t-1}\right]\right)}{\widehat{\sigma}_{g_{M, t-1}}}
\end{array}\right),
\end{aligned}
$$

where $R_{i, t}^{*}$ denotes the return of security $i$ at time $t . g_{i, t-1}$ is the $(M \times 1)$-vector of signals observations. The correlations between the lagged signals and returns in the $(1 \times M)$-vector $\hat{k}$ are called information coefficients (ICs) and measure the signals' informational content. The inverse of the signals' correlation matrix $\widehat{C}^{-1}$ ensures that highly correlated signals have a lower impact on the alpha forecast and vice versa. 
Representing the companies' standardized signal observations, the values in the $(M \times 1)$-vector $z_{i, t-1}$ are referred to as $z$ scores. Because factor investing strategies are based on cross-sectional anomalies, parameter estimation is conducted with regard to cross-sectional observations. Therefore, $\hat{k}$ and $\widehat{C}$ are the same for all securities. This is equivalent to the assumption that a specific stock characteristic incorporates the same informational content and linear co-movement with other signal types for all securities. To obtain ex-ante estimates, we estimate the ICs and $C$ from the time-series averages of their cross-sectional estimates. The applied linear form is strongly related to a Fama-MacBeth regression. Nevertheless, we deviate from the wellknown Fama-MacBeth beta-notation by segregating betas into information coefficients, the characteristics' interaction effects and $z$ scores. By ignoring differences in the firm characteristics' information coefficients and interaction effects, alpha forecasts can be linked directly to the firms' $z$ scores. This naive method of alpha forecasting is frequently applied in practice. However, Heinrich and Zurek (2019) emphasized the important role of ICs in factor investing strategies. Moreover, ICs are one of the main determinants in the fundamental law of active management, which is a simplified framework to explain a portfolio's information ratio. ${ }^{5}$ Therefore, it is straightforward to refrain from using naive alpha forecasts of equally weighted $z$ scores and instead consider the full range of linear interaction effects between the firm characteristics.

Besides alpha forecasts, implementing diversificationbased factor strategies requires the estimation of the residual returns' covariance matrix $\Sigma$. Since the most popular estimator-the sample covariance-leads to high estimation errors, especially in the presence of high-dimensional data, most risk-based investment strategies choose more biased, but less variable estimators for the covariance matrix. We utilize two different covariance estimation methods, which are more stable than the sample estimator $\widehat{\Sigma}_{S}$.

The first method considers the statistical shrinkage procedure applied to a structured target matrix $\widehat{\Sigma}_{T}$,

$\widehat{\Sigma}_{L S}=s \widehat{\Sigma}_{T}+(1-s) \widehat{\Sigma}_{S}$

where the constant $s \in[0,1]$ controls the shrinkage intensity. As a compromise between the highly unstable sample estimator and the highly biased target matrix, the convex combination of both uses the bias-variance trade-off to enhance the out-of-sample performance. Implementing this linear shrinkage method in the context of financial time series requires the target matrix to be chosen with reference to an

\footnotetext{
5 Complementary work on the fundamental law of active management can be found in Clarke et al. (2002), Buckle (2004), Ye (2008) and Ding et al. (2020).
}

assumed correlation structure of the underlying returns. The analysis focuses on the linear shrinkage method of Ledoit and Wolf (2004), which assumes identical pairwise correlations among all $N$ assets and is referred to hereinafter as the Ledoit Wolf constant correlation (LW-CC) estimator. While the variances are kept as their original sample values, the off-diagonal entries of the target matrix are estimated with a constant average sample correlation $\bar{\rho}$. This results in $\widehat{\Sigma}_{T, i j}=\sqrt{\hat{\sigma}_{i} i \hat{\sigma}_{j} j \bar{\rho}}$. For the concrete implementation of this estimation method, we use the optimal shrinkage intensity $s^{*}$, as derived by Ledoit and Wolf (2004).

Finally, to establish a benchmark for the results of combining characteristics- and diversification-based methods, we follow Amenc et al. (2012) and additionally implement a robust factor-based covariance matrix estimator. Using factor models, the covariance matrix of investment returns can be estimated as

$\widehat{\Sigma}_{F M}=B \widehat{\Sigma}_{F} B^{\prime}+\widehat{\Sigma}_{u}$

where $B$ is the matrix of factor loadings, $\Sigma_{F}$ is the sample covariance matrix of the common factors, and $\widehat{\Sigma}_{u}$ is the residual covariance matrix. ${ }^{6}$

Instead of assuming a concrete underlying factor model, as described in Fan et al. (2008), Amenc et al. (2011) choose to use PCA to determine the factors directly from the data. ${ }^{7}$ In data analysis, PCA is a dimension reduction method based on the spectral decomposition of the sample covariance matrix. First introduced by Hotelling (1933), the central idea of PCA is to reduce the dimensionality of a data set and at the same time to retain as much as possible of the present variation between the individual entries (Ledoit and Wolf 2015). In particular, if the first $K$ factors (or sample eigenvectors) govern most of the variability of asset returns, i.e., if $\frac{\sum_{k=0}^{K} \sigma_{F, l}^{2}}{\sum_{k=0}^{N} \sigma_{F, l}^{2}} \approx 1$, then the last $N-K$ factors can be dropped without losing too much information on the underlying covariances. The respective covariance estimator becomes

$\widehat{\Sigma}_{P C A}=V_{K} \widehat{\Lambda}_{K} V_{K}{ }^{\prime}+\hat{\Sigma}_{u}$,

where $V_{K}$ is the $N$-asset by $K$-factor matrix of factor loadings (first $K$ eigenvectors), $\Lambda_{K}$ is the $K \times K$ diagonal matrix of the first $K$ eigenvalues, and $\widehat{\Sigma}_{u}$ is the $N \times N$ diagonal matrix with the sample variances of idiosyncratic components not explained by the first $K$ factors. The application of PCA is

\footnotetext{
${ }^{6}$ Following this definition and assuming $K$ common factors with $K<N$, factor-based methods only need to estimate $K(K+1) / 2$ covariance entries and thus do not suffer from the curse of dimensionality.

7 This approach is widely spread in the recent financial literature, since it does not require a specific factor model, see, e.g., Fan et al. (2013), Fan et al. (2018), Fan et al. (2016).
} 
strongly motivated by the observation that most of the information within a covariance matrix is loaded within the first $K$ largest eigenvalues while the rest of the information is mostly noise. However, determining the number of factors $K$ remains an important question in the literature. ${ }^{8}$

Following Amenc et al. (2011), we refer to the contributions from random matrix theory as in Laloux et al. (2000), Bouchaud and Potters (2009), Bun et al. (2017). In particular, when assuming i.i.d. returns, the eigenvalues of the true random covariance matrix are described by the Marčenko-Pastur (MP) distribution with the upper bound $\lambda_{\max }=\sigma^{2}(1+\sqrt{N / T})^{2}$ and $\sigma^{2}$ corresponding to the average estimated variance across the $N$ assets (Laloux et al. 2000). It is therefore possible to detect which eigenvalues actually carry valuable information and which eigenvalues are random and consist of pure noise. As in Amenc et al. (2011), the number of principal components $K$ for the method in Eq. (9) is set to be the number of eigenvalues with essential information, namely those larger than $\lambda_{\max }$. The described fully data-driven covariance estimation method produces a stable estimator based only on the actually relevant information from the returns and is expected to perform well in a high-dimensional data setting, such as within factor investing strategies.

Instead of explicitly applying covariance estimates with $\widehat{\Sigma}_{L S}$ or $\widehat{\Sigma}_{P C A}$, it is possible to adopt a simplifying structure of the correlation of the residual returns that allows us to rely only on the alpha predictions of the securities. As an alternative to the strong model assumption of $\widehat{\Sigma}_{I}=I$ with $I$ representing the identity matrix, we apply a weaker model assumption on the covariance structure of the residuals $\Omega$ from the complete factor model (2). Within the OOP perspective MacKinlay and Pástor (2000) provide the following relation for the residual covariance matrix $\Sigma$ :

$\Sigma=\alpha \alpha^{\prime} \frac{1}{\left(\frac{\alpha_{\AA}}{\sigma_{\AA}}\right)^{2}}+\Omega$

Here, the expression $\left(\frac{\alpha_{A}}{\sigma_{A}}\right)^{2}$ is the squared information ratio of the optimal orthogonal portfolio and determines its performance contribution to the overall mean variance portfolio. Interestingly, Eq. (10) reveals that with an assumption for $\Omega$ the vector of mispricings is linked to $\Sigma$. By utilizing the Sherman-Morrison formula for the inverse of $\Sigma$, this relation can be inserted in the optimal portfolio weight Eq. (3):
$a_{\mathrm{A}}=\frac{\Omega^{-1} \alpha}{\mathbf{1}_{N}^{\prime} \Omega^{-1} \alpha}$.

It becomes apparent that the residual covariance matrix $\Omega$ from model (2) replaces the covariance matrix $\Sigma$ from model (1). Moreover, under a restricted form for the residual covariance of $\Omega=\sigma^{2} I$ the OOP weights are proportional to the securities' alpha parameters:

$a_{\mathrm{A}}=\frac{\alpha}{1^{\prime} \alpha}$

This result motivates building factor portfolios that exhibit weights, proportional to $\alpha$ and are therefore solely determined by their firm characteristics. ${ }^{9}$ Since securities with the highest alpha forecasts will reach the highest weights, the demonstrated weighting approach is referred to as alpha concentration (Alpha-Con).

The assumption- $\Omega=\sigma^{2} I$-referred to as a "strong form" by MacKinlay and Pástor (2000) might appear very restrictive. However, it is shown empirically and in a simulation that the assumed structure improves the out-of-sample portfolio performance. Since the simple approach of assuming zero-correlated residuals leads to a stable but also biased solution, the question regarding whether the concentrated approach can be advantageous compared to diversified approaches remains open. To study the results within a multifactor setting, we conduct a horse race between the Alpha-Con approach and the two diversification approaches utilizing the LW-CC and PCA estimators, respectively. As an additional alternative to the Alpha-Con approach, we also investigate an equally weighted multifactor portfolio. In the equally weighted multifactor portfolio, the forecasted alphas are applied only in the security selection step, while the number of $P$ selected securities is equally weighted by $1 / P$.

\section{Data}

The S\&P 500 (SPX) and STOXX Europe 600 (STX) indices are used as representative data sets for the US and European stock markets, respectively. Compared to the SPX, the STX comprises different currency areas. To ensure that currency effects do not influence the result, returns are measured in local currency. In particular, the assumption is made that the currencies are hedged, whereby hedging costs are not taken into account. As proxy for the risk free rate, we applied the one-month T-bill rate for the US market and the three-month Euro Government Bond rate for the European market.

\footnotetext{
${ }^{9}$ The Fama-French portfolios are well-known examples of pure characteristics-based factor portfolios.
}

8 There is an extensive line of research on this topic, see, e.g., Bai
and $\mathrm{Ng}(2002)$, Hallin and Liška (2007), Rothman et al. (2010), Fan
et al. (2013). 
Table 1 Mean ICs and bootstrap results of the SPX, and STX sample

\begin{tabular}{|c|c|c|c|c|c|}
\hline \multirow[t]{2}{*}{ Factor } & \multirow[t]{2}{*}{ Characteristic } & \multicolumn{2}{|l|}{ SPX } & \multicolumn{2}{|l|}{ STX } \\
\hline & & Mean & SE & Mean & SE \\
\hline \multirow[t]{5}{*}{ Growth } & Earnings Growth 1 Year (EPSG1Y) & -0.126 & 0.563 & $1.188 * *$ & 0.429 \\
\hline & Earnings Growth 3 Years Average (EPSG3Y) & 0.499 & 0.483 & $0.796 *$ & 0.426 \\
\hline & Internal Growth Rate (IGR) & $1.625^{* *}$ & 0.655 & $1.250 * *$ & 0.496 \\
\hline & Earnings Growth Trend (EPSGT) & $1.853 * *$ & 0.807 & $2.040 * *$ & 0.692 \\
\hline & Sales Growth Trend (SPSGT) & 0.684 & 0.750 & 0.593 & 0.597 \\
\hline \multirow[t]{4}{*}{ Value } & Dividend Yield (DivYld) & 0.781 & 0.887 & $-1.868 * *$ & 0.939 \\
\hline & Earnings to Price (EtP) & -0.546 & 0.984 & -0.866 & 0.785 \\
\hline & Book to Price (BtP) & -1.250 & 1.089 & $-3.504 * *$ & 1.195 \\
\hline & Cash Flow to Enterprise Value (CFOtEV) & 0.247 & 0.829 & 0.393 & 0.609 \\
\hline \multirow[t]{3}{*}{ Quality } & Return on Equity (RoE) & $1.762 * *$ & 0.720 & $2.534 * *$ & 0.706 \\
\hline & Debt to Equity (DtE) & 0.495 & 0.598 & $3.247 * *$ & 0.844 \\
\hline & Earnings Variability (EVar) & 0.472 & 0.558 & 0.283 & 0.455 \\
\hline \multirow[t]{2}{*}{ Momentum } & 6 Months Price Momentum (Pmom6M) & 0.651 & 1.553 & 1.616 & 1.407 \\
\hline & 12 Months Price Momentum (Pmom12M) & 1.529 & 1.435 & $3.251 * *$ & 1.314 \\
\hline \multirow[t]{2}{*}{ Low-Volatility } & Inverse Beta (InvB) & $3.974 * *$ & 1.196 & $3.082 * *$ & 0.941 \\
\hline & Inverse Volatility (InvVola) & $3.301 * *$ & 1.211 & $2.829 * *$ & 1.015 \\
\hline
\end{tabular}

This table shows (in\%) the means and bootstrap standard errors of the 216 IC realizations over the whole time interval of the SPX and STX samples from 100.000 bootstrap resamples. Mean ICs where the confidence interval does not cross zero from the 5 (2.5) and 95 (97.5) percentiles of the $90 \%$ (95\%) confidence interval are market with one (two) asterisks
The dataset ${ }^{10}$ includes firm characteristics and stock returns from the beginning of 2002 until the end of 2019. 2002 was chosen as a starting point, because we observed a higher number of significant predictors of monthly U.S. returns prior to 2002. Due to limited space, we do not consider time-varying predictability. However, we partially address the problem of misspecification of the factor model by also applying factors with a small number of significant predictors. In addition, we have noted a significantly lower data availability for the STX index prior to 2002 .

With value, growth, momentum, quality and low-volatility, our study includes five well-known factors which corresponds to the factor setting of Zurek and Heinrich (2021). Each factor is based on several firm characteristics, with the multifactor portfolio comprising overall 16 firm characteristics. ${ }^{11}$ The factor composition is based on $\mathrm{MSCI}^{12}$ factor portfolios, as these are widely accepted in the industry. In the

\footnotetext{
10 The dataset, including reporting dates, is provided by the Bloomberg database.

11 The examined factors and the corresponding firm characteristics are shown in Table 7 in the appendix.

12 The MSCI Global Investable Market Value and Growth Index Methodology (September 2017), the MSCI Quality Indexes Methodology (June 2017) and the MSCI Momentum Indexes Methodology (June 2017) are used as guidelines. Due to data availability, the factor specification differs in some respects from the MSCI standard, in particular by applying trailing instead of forward data.
}

case of the low-volatility factor, we have chosen to deviate from the MSCI specification. The MSCI Low-Volatility factor uses a minimum variance approach to reduce the overall risk of the portfolio and thus differs from the other factors, which are based purely on firm characteristics. Therefore, the low-volatility factor is applied by using the characteristic-based method of Chow et al. (2014). This approach fits well with the construction methods of the other factors.

For our empirical out-of-sample analysis, we apply daily trailing data observations. This assures that the firm characteristics always resort to the most actual data points and consider the ongoing fluctuations in price-dependent data. Firm characteristics for which a higher $z$ score does not reflect a higher expected return are multiplied by minus one. In our case, this applies to earnings variability and debt to equity. Statistical outliers are adjusted by the method of DeMiguel et al. (2020) by setting equal all observations greater (less) than the defined threshold to the third (first) quartile plus (minus) three times the interquartile range. Zurek and Heinrich (2021) have shown that the Alpha-Con approach tilts the factor exposure towards those factors that exhibit firm characteristics with the highest informational content. Since the diversification approaches can have a disrupting effect on the factor exposures, it is important to compare the ICs of each firm characteristic. Besides ICs, Zurek and Heinrich (2021) also discuss the effects of the cross-sectional 
correlations between the firm characteristics. ${ }^{13}$ However, as the majority of correlation coefficients are small, the overall impact on the alpha forecast is limited. Table 1 shows the time series means of the 216 cross-sectional IC realizations within the selected test period. Bootstrap standard errors (SEs) and 90\% (95\%) confidence intervals are calculated for 100,000 bootstrap resamples to quantify the uncertainties in the inferences. The sample standard deviation of the average ICs across bootstrap samples is used as an estimate of the standard error. Furthermore, the 5\% (2.5\%) and 95\% $(97.5 \%)$ percentiles of the bootstrap samples are calculated for the mean ICs to obtain the lower and upper bounds of the confidence intervals, respectively. The mean ICs where the confidence interval does not cross zero from the 5 (2.5) and 95 (97.5) percentiles of the $90 \%$ (95\%) confidence interval are marked as significant.

The majority of signals show positive mean ICs, which are close to zero. It is striking that the informational content of the individual characteristics varies considerably between the samples. In terms of the number of significant signals within the individual samples, two scenarios with different predictability can be identified. While the SPX exhibits only five signals with significant ICs, in the STX most ICs are significant with a total of 11 signals. Therefore, the SPX multifactor model represents a case in which an investor has identified a moderate number of ex ante predictors, and thus an accurate forecast is unlikely. In contrast, the multifactor model of the STX sample represents a well-defined factor model.

Furthermore, a strong difference in ICs within and between factors can be observed. The low-volatility factor appears to be the only factor with characteristics showing significant positive ICs in both samples. The large differences in IC structure across samples and factors underscore the importance of applying alpha predictions that account for these differences. In addition, it also demonstrates the need to compare strategies in different scenarios to achieve robust results.

\section{Out-of-sample backtest}

The backtest framework is chosen to closely resemble the realistic investment behavior of an institutional investor, with the main objective of outperforming an underlying cap-weighted benchmark portfolio. This objective requires considering weighting constraints, rebalancing costs, commonly used rebalancing frequencies and representative data sets. The results are analyzed for the SPX and STX samples

\footnotetext{
13 Heatmaps of the firm characteristics' cross-sectional correlations are shown in Figs. 2 and 3 in the appendix.
}

Table 2 Weight concentrations of the LO portfolios in the SPX and STX samples

\begin{tabular}{|c|c|c|c|c|c|}
\hline \multirow[t]{2}{*}{$q t^{+}(\%)$} & \multirow[t]{2}{*}{ Method } & \multicolumn{2}{|c|}{ Max. weights } & \multicolumn{2}{|c|}{$\begin{array}{l}\text { Norm. Herfind- } \\
\text { ahl index }\end{array}$} \\
\hline & & SPX & STX & SPX & STX \\
\hline \multirow[t]{4}{*}{90} & Alpha-Con & 4.94 & 6.50 & 0.16 & 0.22 \\
\hline & $1 / N$ & 2.00 & 1.66 & 0.00 & 0.00 \\
\hline & LW-CC & 3.69 & 3.76 & 1.72 & 1.30 \\
\hline & PCA & 3.69 & 3.76 & 1.06 & 0.95 \\
\hline \multirow[t]{4}{*}{80} & Alpha-Con & 3.36 & 4.56 & 0.18 & 0.19 \\
\hline & $1 / N$ & 1.00 & 0.83 & 0.00 & 0.00 \\
\hline & LW-CC & 2.44 & 2.49 & 1.45 & 1.15 \\
\hline & PCA & 2.44 & 2.49 & 0.86 & 0.74 \\
\hline \multirow[t]{4}{*}{70} & Alpha-Con & 2.89 & 3.87 & 0.22 & 0.20 \\
\hline & $1 / N$ & 0.67 & 0.56 & 0.00 & 0.00 \\
\hline & LW-CC & 2.05 & 2.08 & 1.07 & 1.10 \\
\hline & PCA & 2.05 & 2.08 & 0.73 & 0.66 \\
\hline
\end{tabular}

This table shows (in\%) the results of the normalized Herfindahl index and the maximum portfolio weights of the strategies. Both measures are calculated over all weights in the long portfolios across all rebalancing dates

using the multifactor settings described in Sect. 2. In order to create an appropriate comparison with the parent index, the investable securities consist of the securities that are part of the parent index at the time of rebalancing. Regarding the dataset, the backtest uses only point-in-time data, ${ }^{14}$ i.e., only firm characteristics that were available in the database at the current time. For the estimation period, a five-year rolling window, corresponding to 60 monthly observations, is used. The out-of-sample evaluation interval begins in 2007 and ends in 2019. The portfolio is rebalanced at monthly intervals. Trading costs caused by rebalancing are considered by quantifying the cost-relevant volume with the portfolio turnover rate $\left(\mathrm{PTR}_{t}=\sum_{i=1}^{N}\left|a_{i, t-1}-a_{i, t}\right|\right)$. The PTR determines the percentage of the portfolio that causes trading costs. In this context, a PTR of $30 \%$ signifies that $15 \%$ of the old portfolio is sold. Subsequently, the incoming liquidity has to be reinvested, and trading costs are incurred for $30 \%$ of the portfolio. Following Frazzini et al. (2018), the costs are assumed to be ten basis points per traded volume. The calculated costs are demarcated on the day they arise. Consequently, these costs lead to a direct reduction in the return on the rebalancing day.

\footnotetext{
${ }^{14}$ We have not applied any restated data in the estimates, and always used the first reported values.
} 
Table 3 Backtest LO results of the SPX and STX samples

\begin{tabular}{|c|c|c|c|c|c|c|c|}
\hline \multirow[t]{2}{*}{$\overline{q t^{+}(\%)}$} & \multirow[t]{2}{*}{ Method } & \multicolumn{2}{|l|}{ Alpha } & \multicolumn{2}{|c|}{ Tracking error } & \multicolumn{2}{|c|}{ Information ratio } \\
\hline & & SPX (\%) & $\operatorname{STX}(\%)$ & SPX (\%) & STX (\%) & SPX & STX \\
\hline \multirow[t]{4}{*}{90} & Alpha-Con & $4.63^{*}$ & 2.94 & 9.62 & 8.03 & 0.48 & 0.37 \\
\hline & $1 / N$ & $4.70^{*}$ & 3.32 & 9.38 & 7.50 & 0.50 & 0.44 \\
\hline & LW-CC & $5.82 * *$ & $3.94 * *$ & 7.98 & 6.90 & $0.73 *$ & 0.57 \\
\hline & PCA & $5.76 * * *$ & $4.58 * *$ & 7.78 & 6.73 & $0.74 *$ & $0.68 *$ \\
\hline \multirow[t]{4}{*}{80} & Alpha-Con & 2.97 & $3.32 *$ & 8.00 & 6.70 & 0.37 & 0.50 \\
\hline & $1 / N$ & 2.32 & $3.71 * *$ & 7.21 & 6.04 & 0.32 & 0.61 \\
\hline & LW-CC & $4.34 * *$ & $4.71 * * *$ & 6.32 & 5.80 & $0.69 *$ & $0.81 *$ \\
\hline & PCA & $3.11^{* *}$ & $5.59 * * *$ & 5.47 & 5.29 & 0.57 & $1.06^{* *}$ \\
\hline \multirow[t]{4}{*}{70} & Alpha-Con & 2.63 & $3.63 * *$ & 7.42 & 6.18 & 0.35 & 0.59 \\
\hline & $1 / N$ & 1.88 & $4.16 * * *$ & 6.39 & 5.44 & 0.29 & 0.76 \\
\hline & LW-CC & $3.55 * *$ & $4.48 * * *$ & 5.73 & 5.47 & $0.62 *$ & 0.82 \\
\hline & PCA & $2.44 *$ & $4.96 * * *$ & 4.91 & 4.71 & 0.50 & $1.05 * *$ \\
\hline
\end{tabular}

Significant alpha parameters are marked with asterisks. Significant inference in the differences between the information ratios of the Alpha-Con and the diversification methods are likewise marked with asterisks. One, two, or three asterisks represent the significance of the test, i.e., the rejection of the null hypothesis, with a level of probability of $10 \%, 5 \%$, and $1 \%$, respectively

\begin{tabular}{|c|c|c|c|c|c|c|c|}
\hline \multirow[t]{2}{*}{$q t^{-} / q t^{+}(\%)$} & \multirow[t]{2}{*}{ Method } & \multicolumn{2}{|l|}{ Alpha } & \multicolumn{2}{|c|}{ Tracking error } & \multicolumn{2}{|c|}{ Information ratio } \\
\hline & & SPX $(\%)$ & $\operatorname{STX}(\%)$ & $\operatorname{SPX}(\%)$ & $\operatorname{STX}(\%)$ & SPX & STX \\
\hline \multirow[t]{4}{*}{$10 / 90$} & Alpha-Con & $10.40 * *$ & $12.05^{* *}$ & 16.78 & 17.78 & 0.62 & 0.68 \\
\hline & $1 / N$ & $9.07 * *$ & $11.26^{* *}$ & 16.20 & 16.44 & 0.56 & 0.68 \\
\hline & LW-CC & $12.08 * * *$ & $9.51 * *$ & 13.88 & 14.58 & 0.87 & 0.65 \\
\hline & PCA & $11.39 * * *$ & $9.52 * *$ & 13.05 & 13.91 & 0.87 & 0.68 \\
\hline \multirow[t]{4}{*}{$20 / 80$} & Alpha-Con & $7.47 *$ & $9.63 * *$ & 13.89 & 13.66 & 0.54 & 0.70 \\
\hline & $1 / N$ & 5.59 & $8.17 * * *$ & 12.51 & 11.09 & 0.45 & 0.74 \\
\hline & LW-CC & $7.16^{* *}$ & $7.96 * * *$ & 10.75 & 10.86 & 0.67 & 0.73 \\
\hline & PCA & $5.03 *$ & $7.67 * * *$ & 9.21 & 9.83 & 0.55 & 0.78 \\
\hline \multirow[t]{4}{*}{$30 / 70$} & Alpha-Con & $6.56^{*}$ & $8.73 * *$ & 12.44 & 12.06 & 0.53 & 0.72 \\
\hline & $1 / N$ & 4.42 & $6.93 * * *$ & 10.44 & 8.76 & 0.42 & 0.79 \\
\hline & LW-CC & $5.69 * *$ & $7.06^{* * *}$ & 9.27 & 9.67 & 0.61 & 0.73 \\
\hline & PCA & $5.03 *$ & $6.43 * * *$ & 9.21 & 8.20 & 0.55 & 0.78 \\
\hline
\end{tabular}

Significant alpha parameters are marked with asterisks. Significant inference in the differences between the information ratios of the Alpha-Con and the diversification methods are as likewise marked with asterisks. One, two, or three asterisks represent the significance of the test, i.e., the rejection of the null hypothesis, with a level of probability of $10 \%, 5 \%$, and $1 \%$, respectively

\section{Backtest results}

To ensure robust results and to investigate a wide range of portfolio sizes, we apply three different quantiles of $q t^{+}=\{90 \%, 80 \%, 70 \%\}$ in the selection threshold for the long portfolio $\alpha_{q t}^{+}$and $q t^{-}=\{10 \%, 20 \%, 30 \%\}$ in the selection threshold for the short portfolio $\alpha_{q t}^{-}$. Table 2 depicts the corresponding maximum (max.) portfolio weights and the normalized (norm.) Herfindahl index (HI) results for the LO strategies. ${ }^{15}$

Since the unconstrained diversification approaches lead to high weight concentrations, weight constraints. ${ }^{16}$ have been applied within the diversification approaches, to allow for a meaningful comparison with the Alpha-Con approach. The

\footnotetext{
$\overline{15}$ The results for the LS strategies are shown in Table 9 in the appendix.

16 The optimization problem is solved with quadratic programming.
} 
weight bounds are determined by the average max. weights of the Alpha-Con portfolios. Therefore, the max. weights of the diversification approaches are the same for the LW-CC and the PCA diversification approaches. Since the LW-CC and PCA approaches reach the max. weight in almost every rebalancing, the average max. weights of the diversified approaches are nearly identical to those of the Alpha-Con approach.

Almost all weights of the portfolios are below 5\%. Consequently, the strategy weights are in line with the usual weighting rules applied to institutional investment funds. The higher HI values in the diversification approaches indicate that the Alpha-Con portfolios are in fact less concentrated. Moreover, the HI results of the Alpha-Con portfolios are very close to zero, which equals the HI value of the $1 / N$ strategy. This indicates that the predicted alphas of the selected securities in the Alpha-Con portfolios are very similar to each other.

To examine the portfolio performance compared to the associated cap-weighted benchmark portfolios, in Tables 3 and 4, we report three important performance measuresthe annualized alpha parameters, tracking errors, and information ratios for each strategy for the LO portfolios and LS portfolios, respectively. The time series of monthly portfolio returns is used to compute the out-of-sample portfolio alphas, which are estimated from a regression of the portfolio returns against the associated benchmark returns. To determine the strategies' information ratios, the portfolios' alpha parameters are divided by the tracking errors, calculated from the standard deviations of the regression residuals.

Regarding the SPX sample, the only significant alpha performance result within the Alpha-Con portfolios can be observed for the $90 \%$ quantile portfolio. This outcome is not surprising since the SPX alpha forecasting model contains a moderate number of firm characteristics with significant mean ICs. However, it is remarkable that the two diversification approaches achieve significant positive alpha results in all three SPX samples. The alpha concentration approaches of the STX sample obtain significant alphas in the $80 \%$ and $70 \%$ portfolios, which can be explained by the large number of significant predictors. In comparison, the diversification approaches manage to significantly outperform the STX index in all three samples. The qualitative comparison demonstrates that the diversification approaches definitely achieve a higher alpha in all LO portfolios compared to Alpha-Con portfolios.

Due to the low concentrated portfolio weights of the Alpha-Con approach, its performance is similar to the performance of the $1 / N$ portfolios. In the SPX sample, the $1 / N$ approach performs slightly worse, whereas in the STX sample, it performs slightly better. Compared to each other, both diversification approaches lead to similar alpha results.
As a measure of active risk, the tracking errors reveal the extent to which the diversified strategies are able to reduce the portfolio risk. In all samples, the LW-CC and PCA approaches obtain a lower tracking error compared to the Alpha-Con approach. The $1 / N$ portfolios are also able to reduce portfolio risk, but to a lesser extent than the diversification approaches. One can therefore conclude that the diversification approaches indeed reduce portfolio risk. However, in both indices, the tracking error differences between the applied strategies are not significant.

The combination of higher alpha results with lower tracking errors within the diversification approaches leads to much better information ratios compared to the AlphaCon approach. To determine the inference in the differences between the information ratios of the Alpha-Con and the diversification portfolios, a Ledoit and Wolf (2008) test is applied. The examination includes tests for statistically significant differences at the $1 \%, 5 \%$ and $10 \%$ levels. In all samples, at least one diversification approach yields significantly better information ratio results compared to the Alpha-Con portfolios.

Table 4 shows the performance results for the LS portfolios. Due to the short portfolios, the corresponding market risk exposures are reduced, resulting in alphas that are multiple times greater than those of the LO portfolios. With minor exceptions for the $1 / N$ portfolios, in almost all samples the alpha performance results are significantly positive. Especially in comparison to the LO results, it is noticeable that nearly all alphas of the Alpha-Con approach achieve higher values than those in the diversification approaches. Consistent with the LO portfolio results, the tracking errors of the diversification approaches are all smaller compared to the Alpha-Con portfolios.

In contrast to the LO portfolios, the information ratio results of the LS portfolios show very limited differences in the performance between the approaches. Therefore, no significant improvements can be observed for the diversification approaches. The results for the LS portfolios provide reasonable doubt regarding the proposition that diversified portfolio weights lead to overall performance advantages over alpha concentrated portfolio weights.

To analyze these findings in more detail, Tables 5 and 6 present the corresponding average factor exposures of the individual factors for the LO and LS portfolios. The factor exposures are measured by the weighted $z$ scores, consistent with Ghayur et al. (2018). Our results show that the exposure distribution among the individual factors is very heterogeneous. The large factor exposure variation can be mainly explained by the ICs of the characteristics within the factors. For instance, the value factor of the STX sample contains two significantly negative ICs, which causes a negative value factor exposure. In contrast, the value factor of the SPX sample also contains characteristics with negative ICs, but 
Table 5 Average factor exposures of LO strategies

\begin{tabular}{|c|c|c|c|c|c|c|c|}
\hline \multirow{2}{*}{$\begin{array}{l}q t^{+} \\
(\%)\end{array}$} & \multirow[t]{2}{*}{ Factor } & \multirow[t]{2}{*}{ Strategy } & \multicolumn{5}{|c|}{ Factor exposure } \\
\hline & & & Growth (\%) & Value $(\%)$ & Quality (\%) & Momentum (\%) & Low-volatility (\%) \\
\hline \multirow[t]{8}{*}{90} & \multirow[t]{4}{*}{ SPX } & Alpha-Con & 13.87 & 30.01 & -12.55 & 10.26 & 82.59 \\
\hline & & $1 / N$ & 13.81 & 25.96 & -11.84 & 10.47 & 78.18 \\
\hline & & LW-CC & 11.57 & 31.70 & -9.78 & 9.62 & 108.72 \\
\hline & & PCA & 11.82 & 27.96 & -10.13 & 10.90 & 102.09 \\
\hline & \multirow[t]{4}{*}{ STX } & Alpha-Con & 20.96 & -6.78 & 21.26 & 45.69 & 54.72 \\
\hline & & $1 / N$ & 20.68 & -7.21 & 21.02 & 42.89 & 46.12 \\
\hline & & LW-CC & 15.57 & -3.87 & 26.79 & 41.73 & 110.63 \\
\hline & & PCA & 16.23 & -5.25 & 25.85 & 40.11 & 100.48 \\
\hline \multirow[t]{8}{*}{80} & \multirow[t]{4}{*}{ SPX } & Alpha-Con & 13.23 & 20.70 & -9.89 & 10.44 & 67.35 \\
\hline & & $1 / N$ & 13.18 & 14.02 & -8.01 & 10.51 & 56.34 \\
\hline & & LW-CC & 11.28 & 26.36 & -6.42 & 9.50 & 99.64 \\
\hline & & PCA & 13.58 & 19.65 & -5.50 & 11.02 & 77.85 \\
\hline & \multirow[t]{4}{*}{ STX } & Alpha-Con & 19.55 & -7.83 & 19.50 & 39.14 & 41.23 \\
\hline & & $1 / N$ & 18.84 & -8.49 & 18.70 & 34.11 & 29.62 \\
\hline & & LW-CC & 14.82 & -3.36 & 24.82 & 37.50 & 101.54 \\
\hline & & PCA & 14.17 & -4.44 & 22.61 & 35.50 & 88.24 \\
\hline \multirow[t]{8}{*}{70} & \multirow[t]{4}{*}{ SPX } & Alpha-Con & 12.98 & 16.64 & -7.98 & 10.30 & 59.68 \\
\hline & & $1 / N$ & 12.94 & 7.51 & -4.47 & 10.23 & 42.29 \\
\hline & & LW-CC & 10.97 & 24.69 & -5.30 & 10.12 & 95.33 \\
\hline & & PCA & 12.16 & 18.05 & -3.25 & 9.90 & 71.17 \\
\hline & \multirow[t]{4}{*}{ STX } & Alpha-Con & 18.11 & -8.02 & 18.54 & 35.25 & 35.85 \\
\hline & & $1 / N$ & 16.39 & -8.63 & 17.24 & 27.66 & 22.14 \\
\hline & & LW-CC & 14.63 & -2.76 & 23.86 & 35.91 & 96.61 \\
\hline & & PCA & 12.79 & -3.78 & 21.18 & 33.43 & 82.98 \\
\hline
\end{tabular}

Factor exposures are determined by the weighted $z$ scores where the portfolio weights are applied. Averages are calculated from the time series of the cross-sectional results these are closer to zero and not significant. In consequence, the value factor exposure of the SPX sample remains positive. This example shows that an exposure statement can be derived in particular from the significant ICs. Since lowvolatility is one of the factors with the highest mean ICs among the significant firm characteristics, the exposure of this factor is particularly high.

Considering that our alpha forecasting model takes into account the full range of interaction effects between the firm characteristics, in addition to the ICs, the cross-correlations between the characteristics have an impact on the exposures. Momentum and low-volatility consist of two highly correlated characteristics, whereas the correlations of the characteristics within the quality factor are very low and in some cases even negative. While in the case of momentum and low-volatility the characteristics increase their factor exposure, the quality characteristics can cancel each other out and thus reduce the quality exposure. In particular, the low or even negative quality exposure of the SPX sample indicates that a factor with a low number of significant characteristics in combination with uncorrelated firm characteristics is affected by a loss of factor exposure. Although the quality factor exposure of the STX sample is also affected by the low correlation structure, the factor includes two highly significant characteristics. This leads to a higher quality exposure in the STX sample compared to the SPX.

The Alpha-Con approach tilts the factor exposure towards the factors with significant firm characteristics. In contrast, in the diversification approaches, considering the residual covariances leads to disrupting effects on the factor exposures. The disrupting effects can especially be observed in the low-volatility factor. With regard to the results, it is 
Table 6 Average factor exposures of LS strategies

\begin{tabular}{|c|c|c|c|c|c|c|c|}
\hline \multirow{2}{*}{$\begin{array}{l}q t^{-} / q t^{+} \\
(\%)\end{array}$} & \multirow[t]{2}{*}{ Factor } & \multirow[t]{2}{*}{ Strategy } & \multicolumn{5}{|c|}{ Factor exposure } \\
\hline & & & Growth $(\%)$ & Value $(\%)$ & Quality (\%) & Momentum (\%) & Low-volatility (\%) \\
\hline \multirow[t]{8}{*}{$10 / 90$} & \multirow[t]{4}{*}{ SPX } & Alpha-Con & 48.26 & 13.59 & 14.86 & 40.52 & 139.87 \\
\hline & & $1 / N$ & 44.28 & 11.05 & 11.75 & 37.53 & 132.32 \\
\hline & & LW-CC & 39.96 & 25.84 & 1.87 & 30.21 & 128.54 \\
\hline & & PCA & 39.37 & 24.77 & 1.06 & 29.63 & 122.63 \\
\hline & \multirow[t]{4}{*}{ STX } & Alpha-Con & 53.12 & -47.17 & 87.53 & 96.67 & 105.62 \\
\hline & & $1 / N$ & 50.24 & -44.11 & 82.04 & 90.72 & 92.18 \\
\hline & & LW-CC & 46.65 & -24.88 & 72.74 & 80.65 & 124.15 \\
\hline & & PCA & 45.95 & -24.06 & 68.04 & 78.00 & 112.30 \\
\hline \multirow[t]{8}{*}{$20 / 80$} & \multirow[t]{4}{*}{ SPX } & Alpha-Con & 39.66 & 10.26 & 7.68 & 33.05 & 115.88 \\
\hline & & $1 / N$ & 34.32 & 6.60 & 3.35 & 28.68 & 99.38 \\
\hline & & LW-CC & 33.12 & 27.70 & -6.36 & 19.58 & 102.02 \\
\hline & & PCA & 34.02 & 24.58 & -6.71 & 19.47 & 80.57 \\
\hline & \multirow[t]{4}{*}{ STX } & Alpha-Con & 46.38 & -37.99 & 68.78 & 80.75 & 78.43 \\
\hline & & $1 / N$ & 41.64 & -31.64 & 57.15 & 69.44 & 57.92 \\
\hline & & LW-CC & 38.93 & -14.80 & 53.37 & 63.13 & 94.69 \\
\hline & & PCA & 35.40 & -13.50 & 44.89 & 59.14 & 77.76 \\
\hline \multirow[t]{8}{*}{$30 / 70$} & \multirow[t]{4}{*}{ SPX } & Alpha-Con & 35.71 & 9.46 & 4.83 & 29.47 & 102.77 \\
\hline & & $1 / N$ & 29.34 & 4.80 & 0.54 & 23.85 & 77.49 \\
\hline & & LW-CC & 30.34 & 29.10 & -9.42 & 15.40 & 88.93 \\
\hline & & PCA & 29.48 & 26.13 & -9.32 & 13.46 & 63.45 \\
\hline & \multirow[t]{4}{*}{ STX } & Alpha-Con & 41.98 & -33.31 & 60.07 & 71.35 & 67.24 \\
\hline & & $1 / N$ & 34.56 & -24.21 & 44.03 & 54.51 & 41.99 \\
\hline & & LW-CC & 36.45 & -11.41 & 47.53 & 56.47 & 83.96 \\
\hline & & PCA & 30.82 & -10.23 & 38.19 & 51.86 & 65.10 \\
\hline
\end{tabular}

Factor exposures are determined by the weighted $z$ scores where the portfolio weights are applied. Averages are calculated from the time series of the cross-sectional results evident that the low-volatility factor exposure of the diversification approaches in the LO portfolios is on average almost twice as high as in the Alpha-Con portfolios. This outcome can be explained by the fact that the low-volatility stocks in the LO portfolios are weighted higher by taking covariances into account. As a result, an increasing effect on the low-volatility factor exposure occurs. In the case of the STX and SPX indices, the low-volatility factor is represented by firm characteristics with high informational content. Since the informational content describes the relationship between firm characteristics and excess returns, it is a measure of the predictive power of a firm characteristic. Higher exposures in firm characteristics with high informational content lead to a higher ability to generate alpha. As a result, LO diversified portfolios benefit from both performance drivers, additional alpha and lower risk.
In the short portfolios of the LS strategies, stocks with low-volatility are also weighted higher because of diversification effects. However, since in the LS portfolio the short portfolio is subtracted from the long portfolio, the low-volatility stocks obtain higher negative weights. Therefore, in the LS strategies, the increasing low-volatility exposure effect of the LO portfolios is canceled out between the long and the short portfolios. This canceling effect leads to a similar low-volatility exposure of the diversification approaches and the Alpha-Con portfolios. As a result, compared to the Alpha-Con approach, the diversified factor portfolios achieve smaller alpha performance values than in the LO results. Without the additional alpha performance, in the LS portfolios, the performance results of the diversification approaches are not significantly different compared to the Alpha-Con approach. 
STX Multifactor - LO 80\%

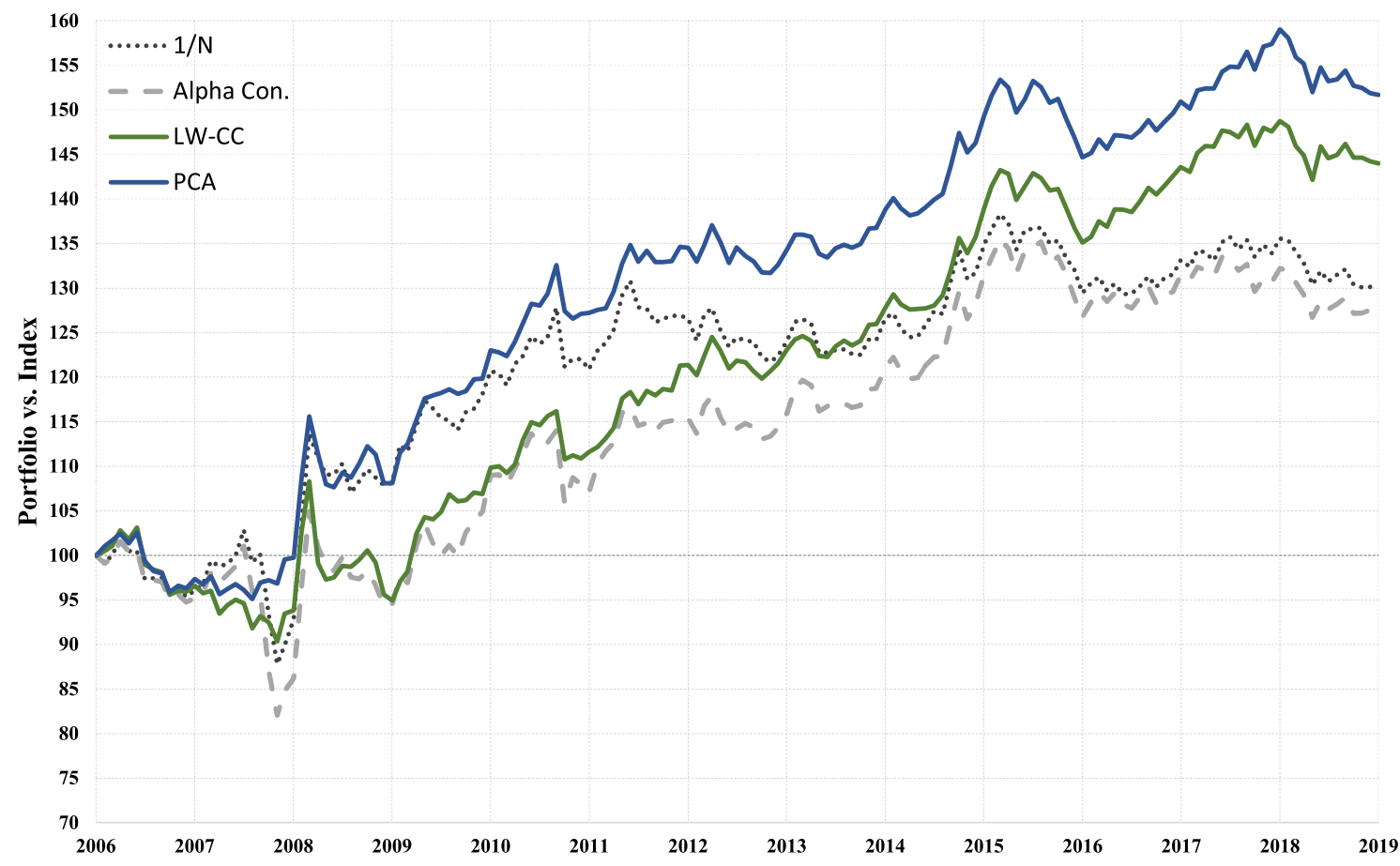

STX Multifactor - LS 20\%/80\%

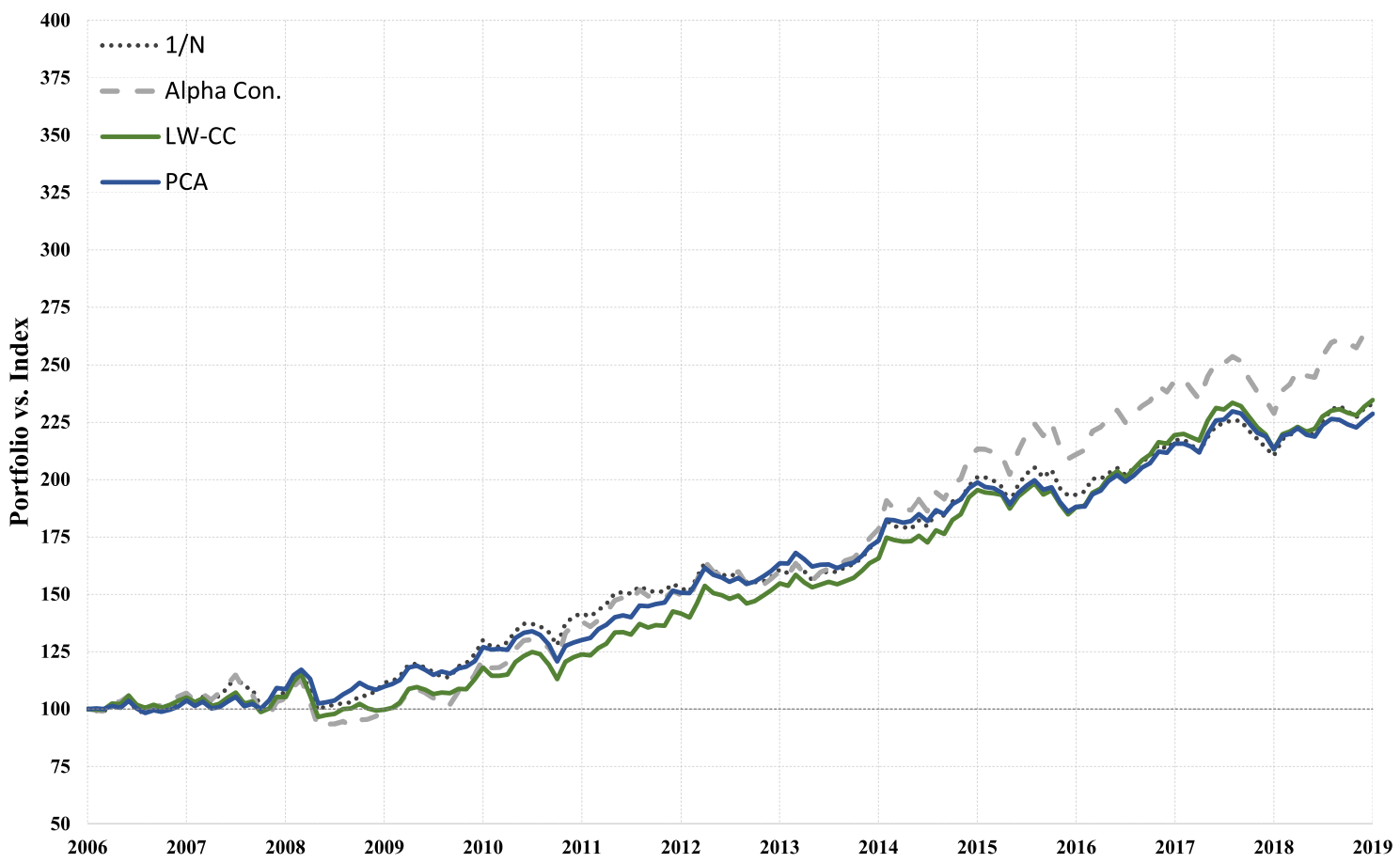

Fig. 1 Relative strength results of long-only $\left(q t^{+}=80 \%\right)$ and long-short $\left(q t^{-}=20 \%, q t^{+}=80 \%\right)$ STX multifactor portfolios. The relative strength results of all approaches in relation to their underlying indices are displayed 
Our empirical results reveal that the Alpha-Con approach is an efficient multifactor strategy. Therefore, we are able to confirm the findings of MacKinlay and Pástor (2000), which present the strong form assumption for the residual covariance matrix $\Omega=\sigma^{2} I$ to be an appropriate assumption. Besides the consideration of ICs within the alpha forecast model, an additional value driver of the Alpha-Con approach can also be identified by its deconcentrated portfolio weights, which are similar to the $1 / N$ portfolio. Although deconcentration is not as efficient as diversification, we can verify the "naive" weighting approach to be a reasonable alternative to the more complex diversification methods.

According to the diversification approaches, at least for the LO portfolios, performance improvements in comparison to the Alpha-Con approach can be observed. However, the alpha performance benefits of the diversification approaches depend to a large extent on the informational content of the firm characteristics in the low-volatility factor. In the case of the STX and SPX indices, the low-volatility factor is represented by firm characteristics with high informational content. The LO portfolios benefit from the diversification approach because the additional low-volatility exposure contributes significantly to higher alpha performance results. In contrast, the LS diversified portfolios are not able to increase the low-volatility exposure relative to the AlphaCon approach, and consequently, the performance results are not significantly different.

Figure 1 depicts the relative strength result charts for the STX $80 \%$ quantile LO portfolios and the 20\%/80\% quantile LS portfolios. ${ }^{17}$ Relative strength is calculated as the ratio of price changes based on the log returns of the strategy portfolios compared to the benchmark portfolios, ${ }^{18}$ scaled to an initial value of 100 . The LW-CC and PCA diversification approaches are represented by blue and green lines, respectively, whereas the Alpha-Con approach and the $1 / \mathrm{N}$ approach are depicted by dashed and dotted lines, respectively. The charts for the LO portfolios show that the LW-CC and PCA diversification approaches achieve higher relative strength compared to the Alpha-Con and 1/N approaches. For the $80 \%$ quantiles, we observe that the PCA approach performs better than the LW-CC approach. However, this observation is not confirmed in the results for the other quantiles. Compared to the LO portfolios, there is no particular difference between the strategies in the LS portfolios. The visualized results further confirm our performance results from Tables 3 and 4.

\footnotetext{
17 The charts for the other quantiles are shown in Figs. 4, 5, 6, 7 and 8 in the appendix.

18 Corresponding to the alpha performance measure, we use riskadjusted returns. Therefore, the benchmark returns are multiplied with the strategies' beta factors.
}

\section{Conclusion}

This article contributes to the current literature on the advantages of diversified versus concentrated multifactor portfolios. We present factor investing from the optimal orthogonal portfolio perspective and apply a linear alpha forecasting model with the extension of two efficient and popular covariance estimation methodologies. Our empirical findings indicate that a pure characteristics-based approach is an efficient multifactor strategy. Moreover, the additional application of the residual covariance matrix improves the out-of-sample performance in long-only strategies. The high informational content of the firm characteristics in the low-volatility factor represents a relevant advantage for diversified approaches. Since informational content is a measure of the predictive power of a firm characteristic, higher exposures to firm characteristics with high informational content lead to a higher ability to generate alpha. In long-only portfolios, diversification strategies are able to provide additional exposure to the low-volatility factor, leading to an increase in excess returns and a reduction in portfolio risk. Regarding the long-short portfolios, the additional low-volatility exposure from the long-only portfolios is canceled out due to a higher weight of low-volatility stocks within the short portfolio. Without the additional low-volatility factor exposure, the performance difference between the pure firm-characteristic-based approach and the diversification approaches is not significant.

Our findings exhibit important practical implications. Since in diversification approaches, the informational content of the low-volatility factor represents an essential performance determinant, it is important to assess the informational content of low-volatility firm characteristics in the respective stock markets. In markets where low-volatility is a factor with significant informational content, increasing exposure to the low-volatility factor provides the opportunity of performance benefits. Furthermore, the performance comparison between long-only and long-short strategies underlines the relevance for the investigation of diversification approaches in terms of factor exposure. In this context, the allocation of exposure to the relevant factors is a fundamental source of portfolio performance. Therefore, it is also essential to use an alpha forecasting approach that is able to consider differences in informational content. A wellcalibrated alpha forecasting model represents the foundation for efficient stock selection and an appropriate portfolio weighting strategy.

\section{Appendix}

See Tables 7, 8 and 9 and Figs. 2, 3, 4, 5, 6, 7 and 8. 
Table 7 Overview: factor strategies and firm characteristics

\begin{tabular}{|c|c|c|}
\hline Factor & Characteristic & Definition \\
\hline \multirow[t]{5}{*}{ Growth } & Earnings Growth 1 Year (EPSG1Y) & EPSG1Y $_{t}=$ YoY EPS Growth ${ }_{t}$ \\
\hline & Earnings Growth 3 Years Average (EPSG3Y) & EPSG3Y $_{t}=\frac{1}{3} \sum_{t=1}^{3}$ YoY EPS Growth \\
\hline & Internal Growth Rate (IGR) & $\mathrm{IGR}_{t}=\frac{\mathrm{TTM} \mathrm{EPS}_{t}}{\mathrm{BVPS}}-\frac{\mathrm{TTM} \mathrm{DPS}_{t}}{\mathrm{BVPS}}$ \\
\hline & Earnings Growth Trend (EPSGT) & $\mathrm{EPSGT}_{t}=\frac{\beta_{E P S}}{1 / 5 \sum_{t=1}^{S}\left|\mathrm{EPS}_{t}\right|}$ \\
\hline & Sales Growth Trend (SPSGT) & $\mathrm{SPSGT}_{t}=\frac{\beta_{S P S}}{1 / 5 \sum_{t=1}^{5}\left|\mathrm{SPS}_{t}\right|}$ \\
\hline \multirow[t]{4}{*}{ Value } & Dividend Yield (DivYld) & $\operatorname{DivYld}_{t}=\frac{\text { TTM DPS }_{t}}{\mathrm{P}_{t}}$ \\
\hline & Earnings to Price (EtP) & $\mathrm{EtP}_{t}=\frac{\mathrm{TTM} \mathrm{EPS}_{t}}{\mathrm{P}_{t}}$ \\
\hline & Book to Price (BtP) & $\mathrm{BtP}_{t}=\frac{\mathrm{BVPS}_{t}}{\mathrm{P}_{t}}$ \\
\hline & Cash Flow to Enterprise Value (CFOtEV) & $\mathrm{CFOtEV}_{t}=\frac{\mathrm{TTM} \mathrm{CFO}_{t}}{\mathrm{EV}_{t}}$ \\
\hline \multirow[t]{3}{*}{ Quality } & Return on Equity (RoE) & $\mathrm{RoE}_{t}=\frac{\mathrm{TTM} \mathrm{EPS}_{t}}{\mathrm{BVPS}_{t}}$ \\
\hline & Debt to Equity (DtE) & $\mathrm{DtE}_{t}=\frac{\mathrm{TD}_{t}}{\mathrm{BV}_{t}}$ \\
\hline & Earnings Variability (EVar) & 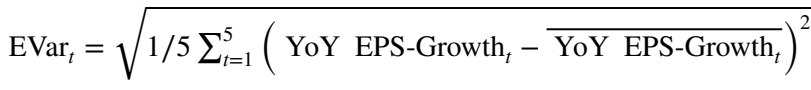 \\
\hline \multirow[t]{2}{*}{ Momentum } & 6 Months Price Momentum (Pmom6M) & n-Months Price Momentum ${ }_{t}=\frac{\left(\frac{P_{t-1}}{P_{t-n-1}}-1\right)-R_{f, t}}{\sigma_{m o m, t}}$ \\
\hline & 12 Months Price Momentum (Pmom12M) & \\
\hline \multirow[t]{2}{*}{ Low-Volatility } & Inverse Beta (InvB) & $\operatorname{InvB}_{t}=\frac{1}{\beta_{L V, t}}$ \\
\hline & Inverse Volatility (InvVola) & InvVola $_{t}=\frac{1}{\sigma_{L V, t}}$ \\
\hline
\end{tabular}

With: Share Price (P), Enterprise Value (EV), Total Debt (TD), Book Value (BV), Book Value per Share (BVPS), Earnings per Share (EPS), Sales per Share (SPS), Dividend per Share (DPS), Cash Flow from Operations (CFO), Year over Year (YoY), Trailing 12 Months (TTM). $\sigma_{m o m, t}$ is the annualized volatility of weekly returns over last 3 years. The volatility $\sigma_{L V, t}$ and the CAPM beta $\beta_{L V, t}$ in the low-volatility factor are calculated using five-year daily data. For the calculation of EPSGT and SPSGT the last 5 yearly restated EPS and SPS are used. $\beta_{E P S}$ and $\beta_{S P S}$ represent slope coefficients from regressions of yearly EPS and SPS observations against the number of months in the 5 year observation interval. For a calculation example of EPSGT and SPSGT see the MSCI Global Investable Market Value and Growth Index Methodology (p. 32, 2017) 
Table 8 Confidence interval results of the bootstrap IC means

\begin{tabular}{|c|c|c|c|c|c|c|c|c|c|}
\hline \multirow[t]{2}{*}{ Factor } & \multirow[t]{2}{*}{ Characteristic } & \multicolumn{4}{|l|}{ SPX } & \multicolumn{4}{|l|}{ STX } \\
\hline & & 0.05-Qt. & 0.95-Qt. & $0.025-\mathrm{Qt}$. & 0.975-Qt. & 0.05-Qt. & 0.95-Qt. & $0.025-\mathrm{Qt}$ & $0.975-\mathrm{Qt}$. \\
\hline \multirow[t]{5}{*}{ Growth } & EPSG1Y & -1.045 & 0.807 & -1.217 & 0.988 & 0.481 & 1.891 & 0.343 & 2.023 \\
\hline & EPSG3Y & -0.296 & 1.293 & -0.447 & 1.445 & 0.093 & 1.495 & -0.041 & 1.620 \\
\hline & IGR & 0.541 & 2.698 & 0.334 & 2.908 & 0.430 & 2.066 & 0.280 & 2.217 \\
\hline & EPSGT & 0.519 & 3.184 & 0.257 & 3.429 & 0.897 & 3.176 & 0.674 & 3.392 \\
\hline & SPSGT & -0.555 & 1.915 & -0.788 & 2.153 & -0.393 & 1.572 & -0.585 & 1.759 \\
\hline \multirow[t]{4}{*}{ Value } & DivYld & -0.685 & 2.230 & -0.969 & 2.514 & -3.398 & -0.325 & -3.707 & -0.029 \\
\hline & EtP & -2.174 & 1.068 & -2.485 & 1.378 & -2.155 & 0.431 & -2.401 & 0.669 \\
\hline & $\mathrm{BtP}$ & -3.043 & 0.538 & -3.389 & 0.880 & -5.451 & -1.529 & -5.821 & -1.147 \\
\hline & CFOtEV & -1.119 & 1.613 & -1.376 & 1.865 & -0.606 & 1.392 & -0.803 & 1.583 \\
\hline \multirow[t]{3}{*}{ Quality } & RoE & 0.575 & 2.938 & 0.334 & 3.158 & 1.367 & 3.697 & 1.147 & 3.919 \\
\hline & DtE & -0.479 & 1.482 & -0.664 & 1.679 & 1.856 & 4.632 & 1.595 & 4.891 \\
\hline & EVar & -0.447 & 1.388 & -0.628 & 1.560 & -0.465 & 1.032 & -0.610 & 1.178 \\
\hline \multirow[t]{2}{*}{ Momentum } & Pmom6M & -1.890 & 3.217 & -2.386 & 3.703 & -0.707 & 3.916 & -1.143 & 4.370 \\
\hline & Pmom12M & -0.824 & 3.889 & -1.277 & 4.347 & 1.089 & 5.397 & 0.667 & 5.820 \\
\hline \multirow[t]{2}{*}{ Low-Volatility } & InvB & 2.005 & 5.935 & 1.629 & 6.303 & 1.522 & 4.614 & 1.220 & 4.910 \\
\hline & InvVola & 1.301 & 5.284 & 0.923 & 5.655 & 1.146 & 4.487 & 0.823 & 4.805 \\
\hline
\end{tabular}

This table shows (in\%) the 5 (2.5) and 95 (97.5) percentiles of the $90 \%$ (95\%) confidence interval of the bootstrap IC means

Table 9 Weight concentrations of the LS portfolios in the SPX and STX samples

\begin{tabular}{|c|c|c|c|c|c|}
\hline \multirow[t]{2}{*}{$q t^{-} / q t^{+}(\%)$} & \multirow[t]{2}{*}{ Method } & \multicolumn{2}{|c|}{$\begin{array}{l}\text { Norm. Herfindahl } \\
\text { index }\end{array}$} & \multicolumn{2}{|c|}{ Max. weights } \\
\hline & & SPX & STX & SPX & STX \\
\hline \multirow[t]{4}{*}{$10 / 90$} & Alpha-Con & 0.16 & 0.17 & 5.73 & 6.50 \\
\hline & $1 / N$ & 0.00 & 0.00 & 2.00 & 1.66 \\
\hline & LW-CC & 1.58 & 1.21 & 3.78 & 3.40 \\
\hline & PCA & 1.21 & 1.02 & 3.78 & 3.40 \\
\hline \multirow[t]{4}{*}{$20 / 80$} & Alpha-Con & 0.16 & 0.16 & 3.59 & 4.56 \\
\hline & $1 / N$ & 0.00 & 0.00 & 1.00 & 0.84 \\
\hline & LW-CC & 1.30 & 1.02 & 2.45 & 2.24 \\
\hline & PCA & 1.17 & 0.73 & 2.45 & 2.24 \\
\hline \multirow[t]{4}{*}{$30 / 70$} & Alpha-Con & 0.19 & 0.18 & 2.94 & 3.87 \\
\hline & $1 / N$ & 0.00 & 0.00 & 0.67 & 0.56 \\
\hline & LW-CC & 1.05 & 0.95 & 2.01 & 1.85 \\
\hline & PCA & 0.76 & 0.61 & 2.01 & 1.85 \\
\hline
\end{tabular}

This table shows (in\%) the maximum weights for the portfolio weights of the strategies and the according results of the normalized Herfindahl index. Both measures are calculated over all weights in the long and short portfolios across all rebalancing dates 
Fig. 2 Average correlations between firm characteristics in the SPX sample

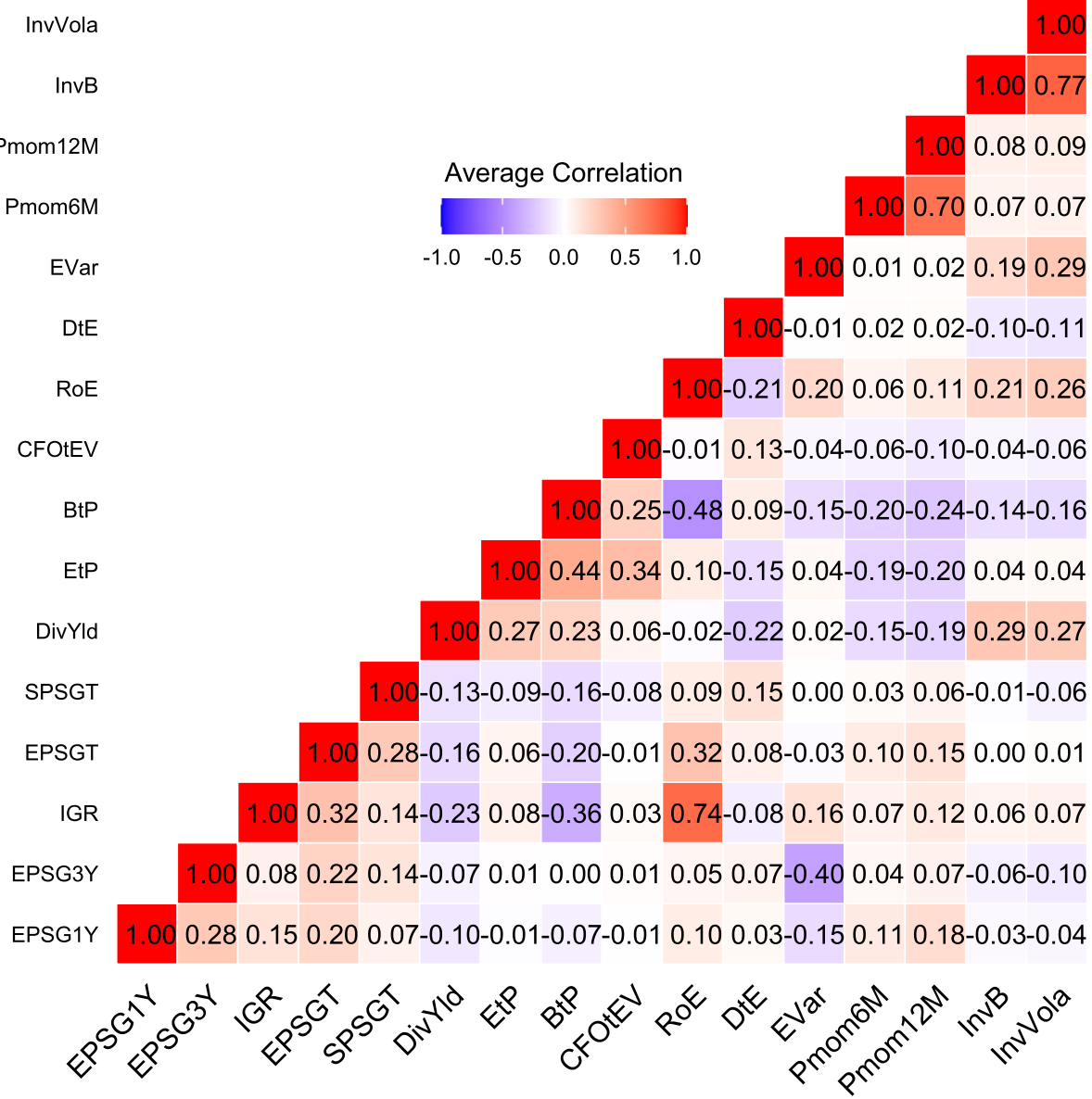


Fig. 3 Average correlations between firm characteristics in the STX sample

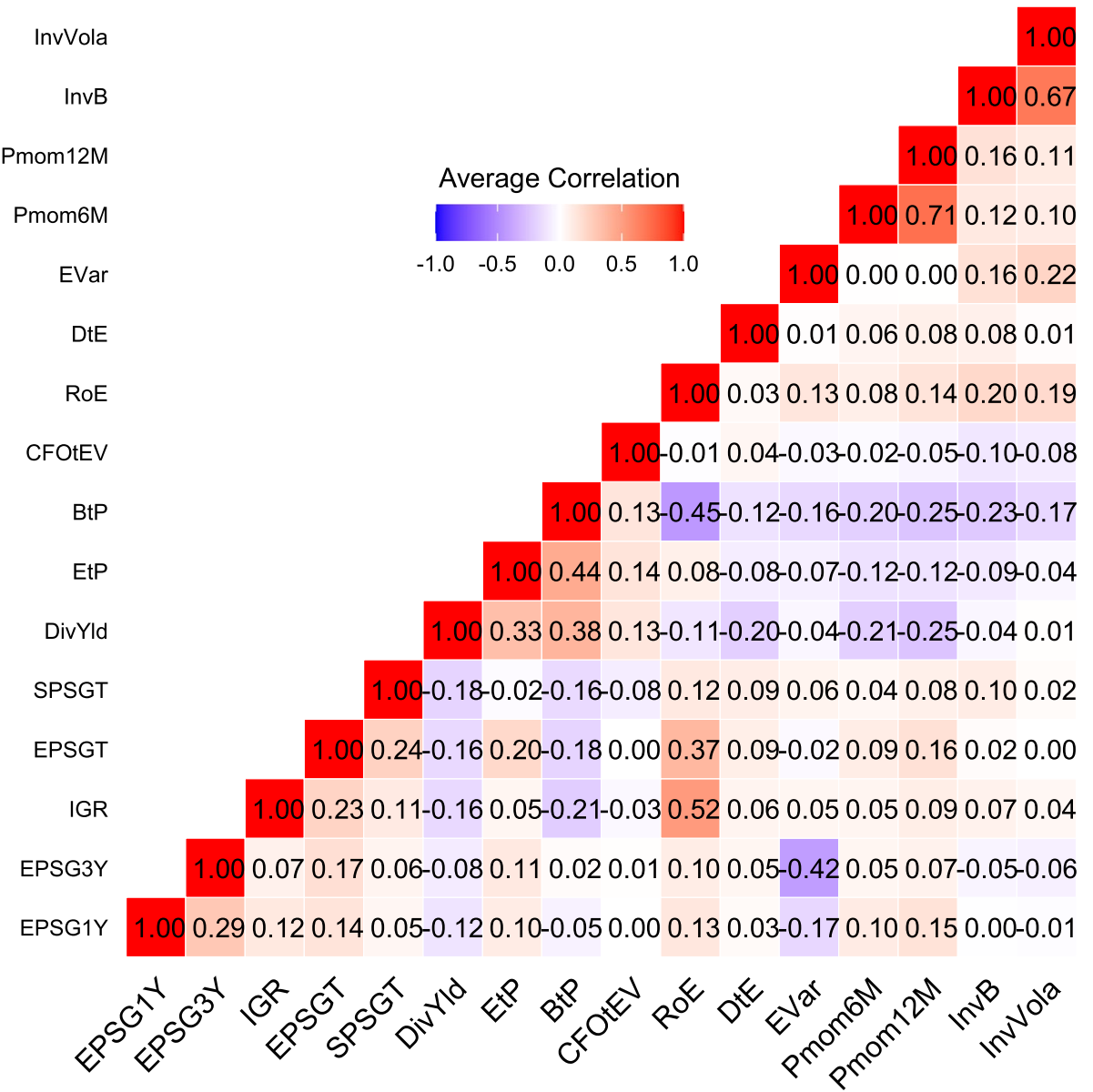


SPX Multifactor - LO 90\%

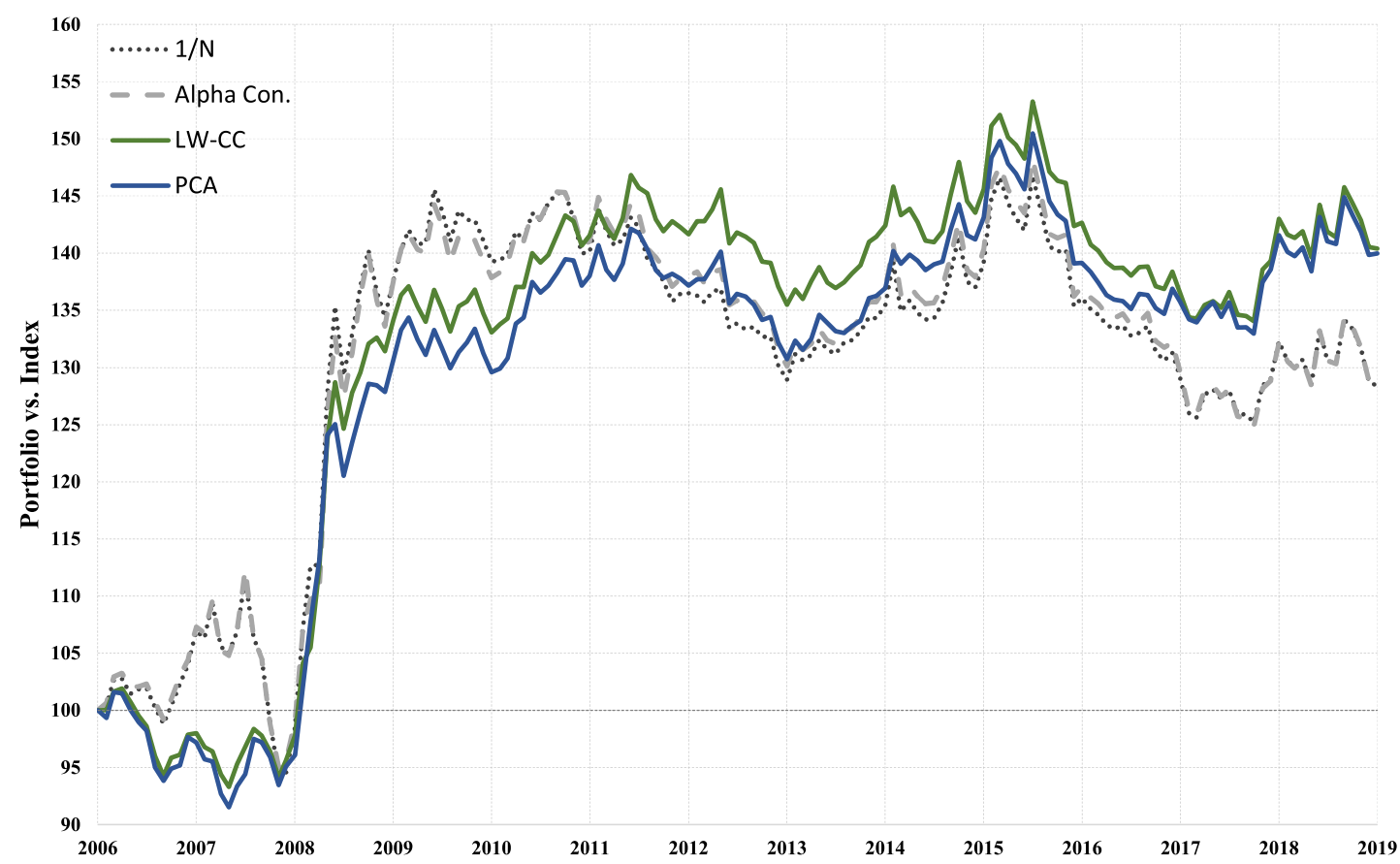

SPX Multifactor - LS 10\%/90\%

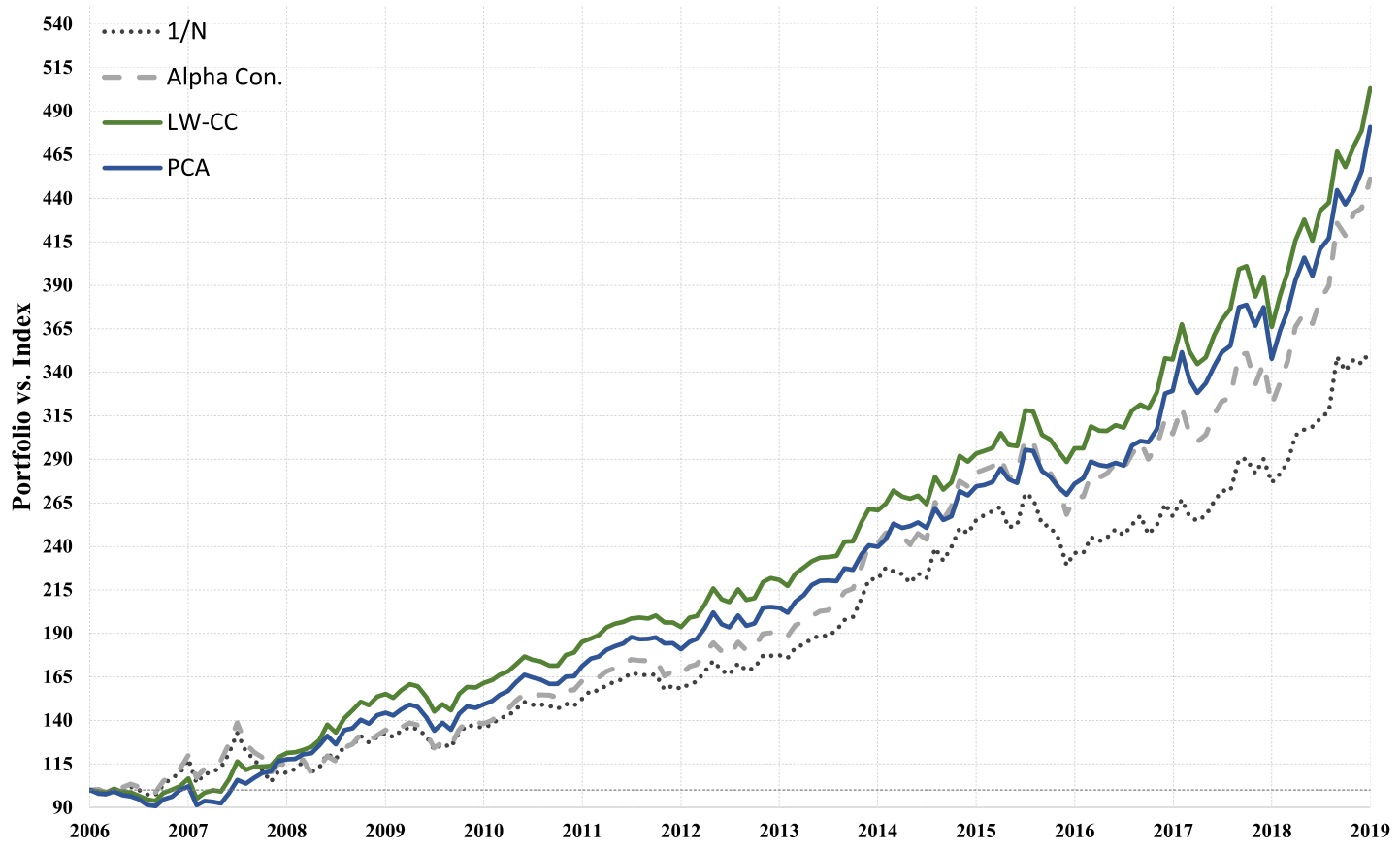

Fig. 4 Relative strength results of long-only $\left(q t^{+}=90 \%\right)$ and long-short $\left(q t^{-}=10 \%, q t^{+}=90 \%\right)$ SPX multifactor portfolios. The relative strength results of all approaches in relation to their underlying indices are displayed 


\section{STX Multifactor - LO 90\%}

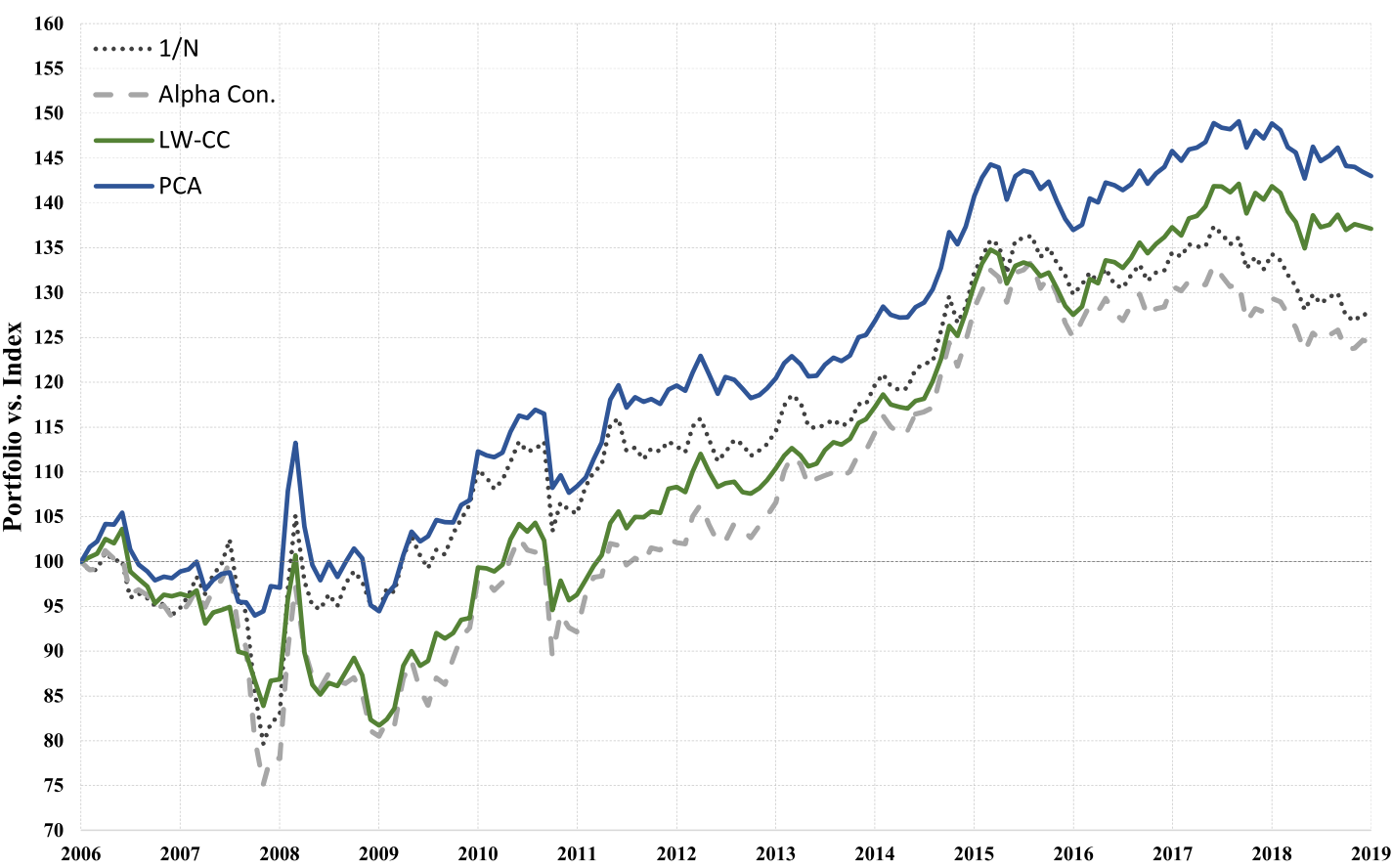

STX Multifactor - LS 10\%/90\%

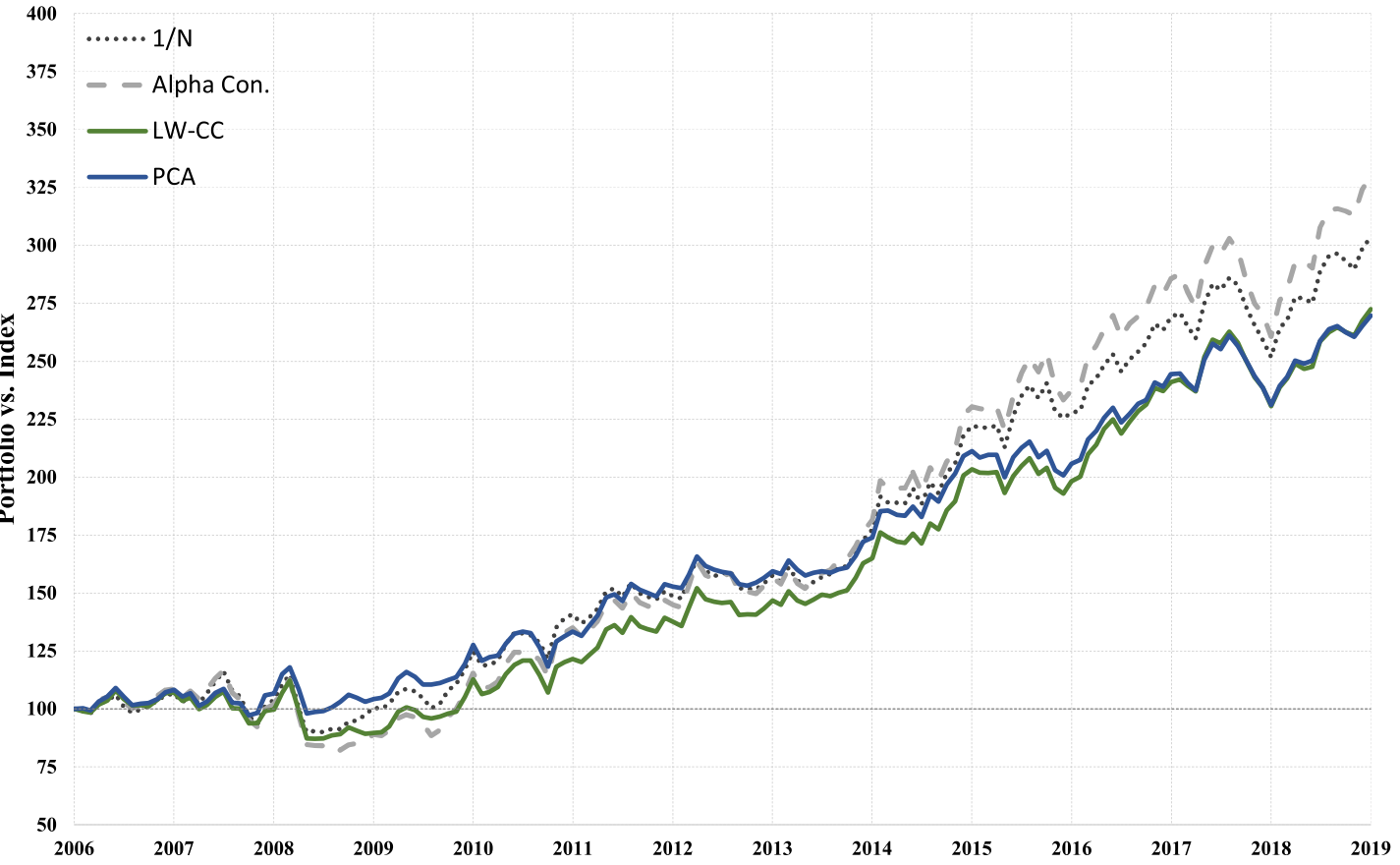

Fig. 5 Relative strength results of long-only $\left(q t^{+}=90 \%\right)$ and long-short $\left(q t^{-}=10 \%, q t^{+}=90 \%\right)$ STX multifactor portfolios. The relative strength results of all approaches in relation to their underlying indices are displayed 


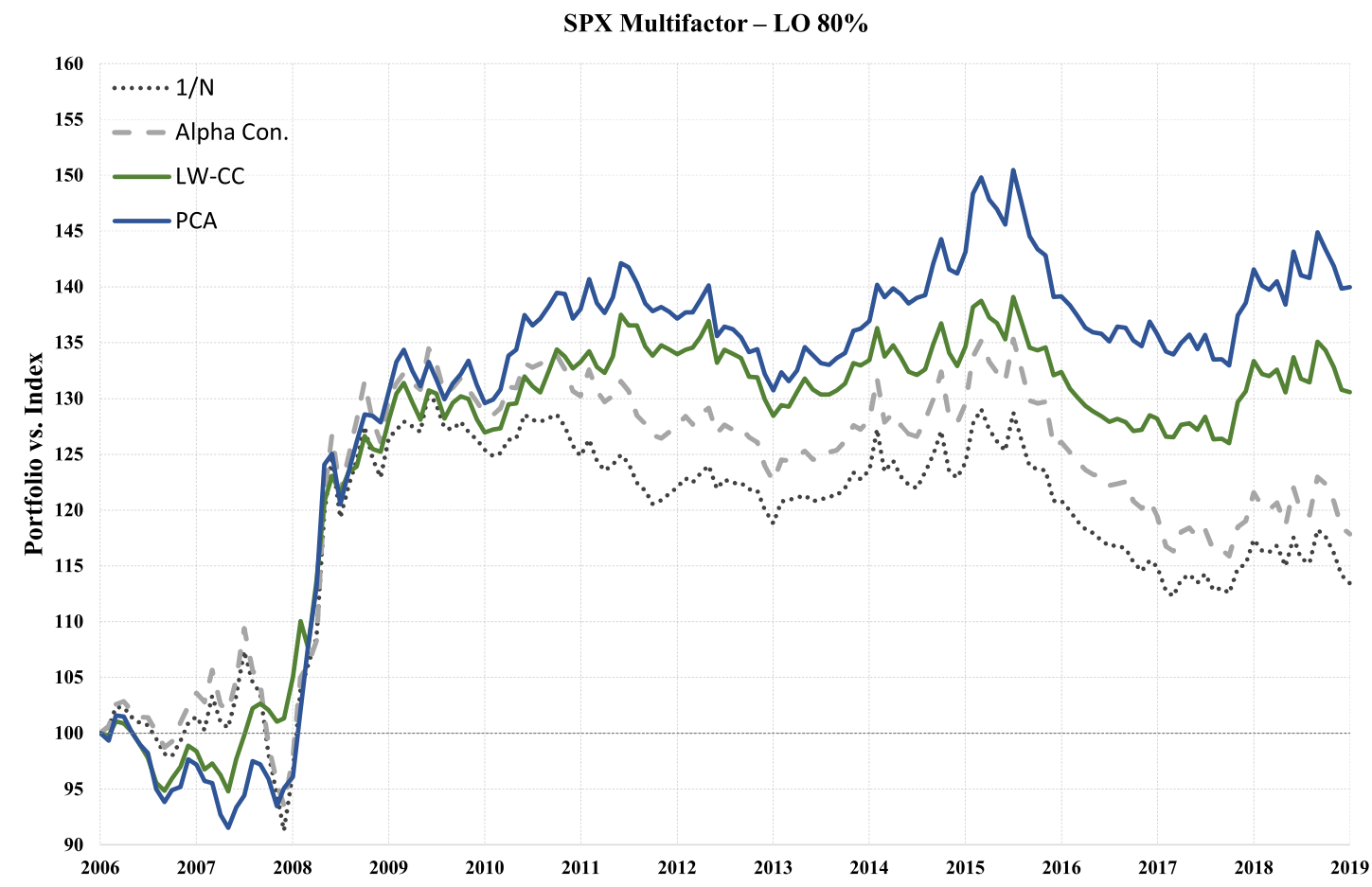

SPX Multifactor - LS 20\%/80\%

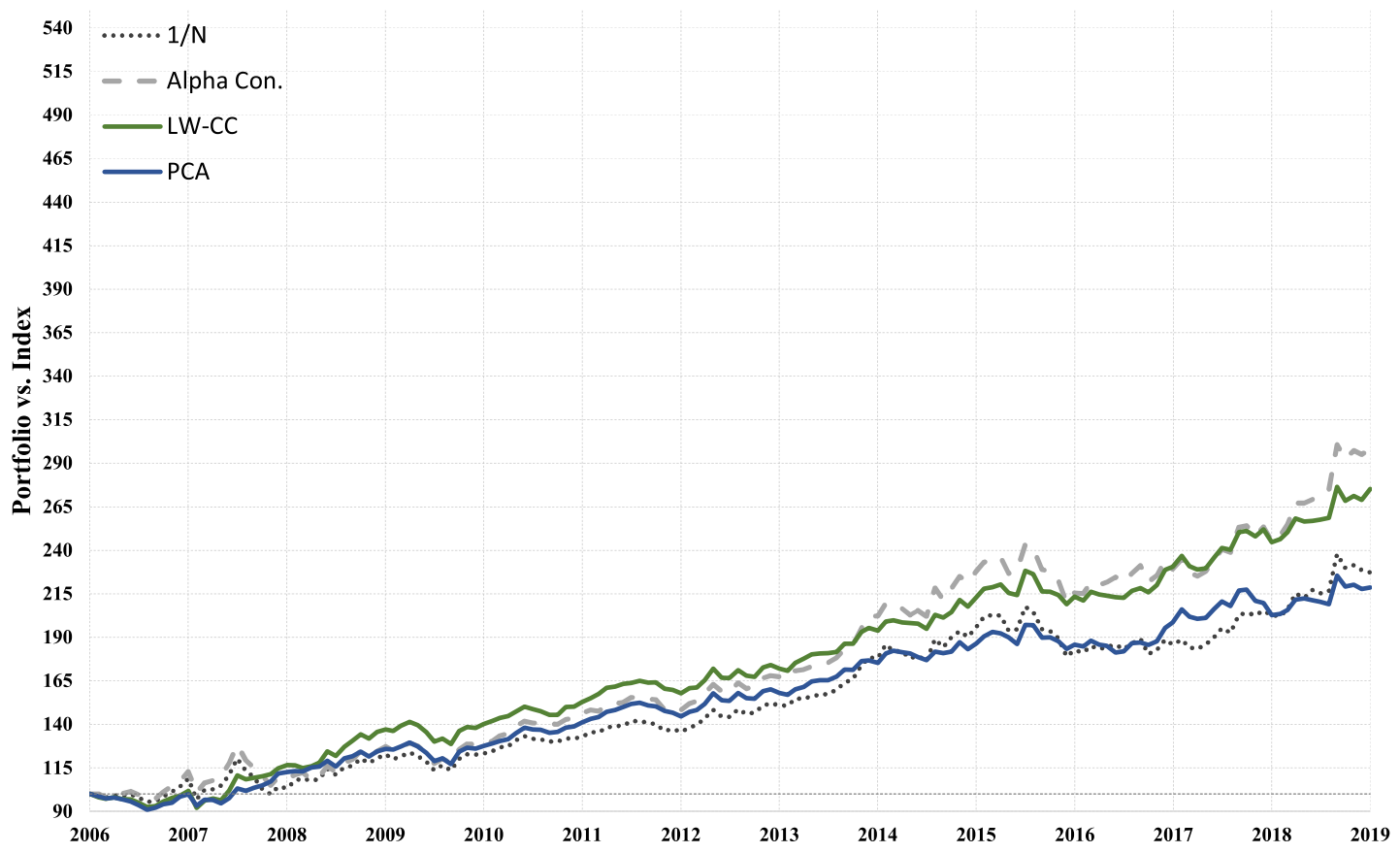

Fig. 6 Relative strength results of long-only $\left(q t^{+}=80 \%\right)$ and long-short $\left(q t^{-}=20 \%, q t^{+}=80 \%\right)$ SPX multifactor portfolios). The relative strength results of all approaches in relation to their underlying indices are displayed 
SPX Multifactor - LO 70\%

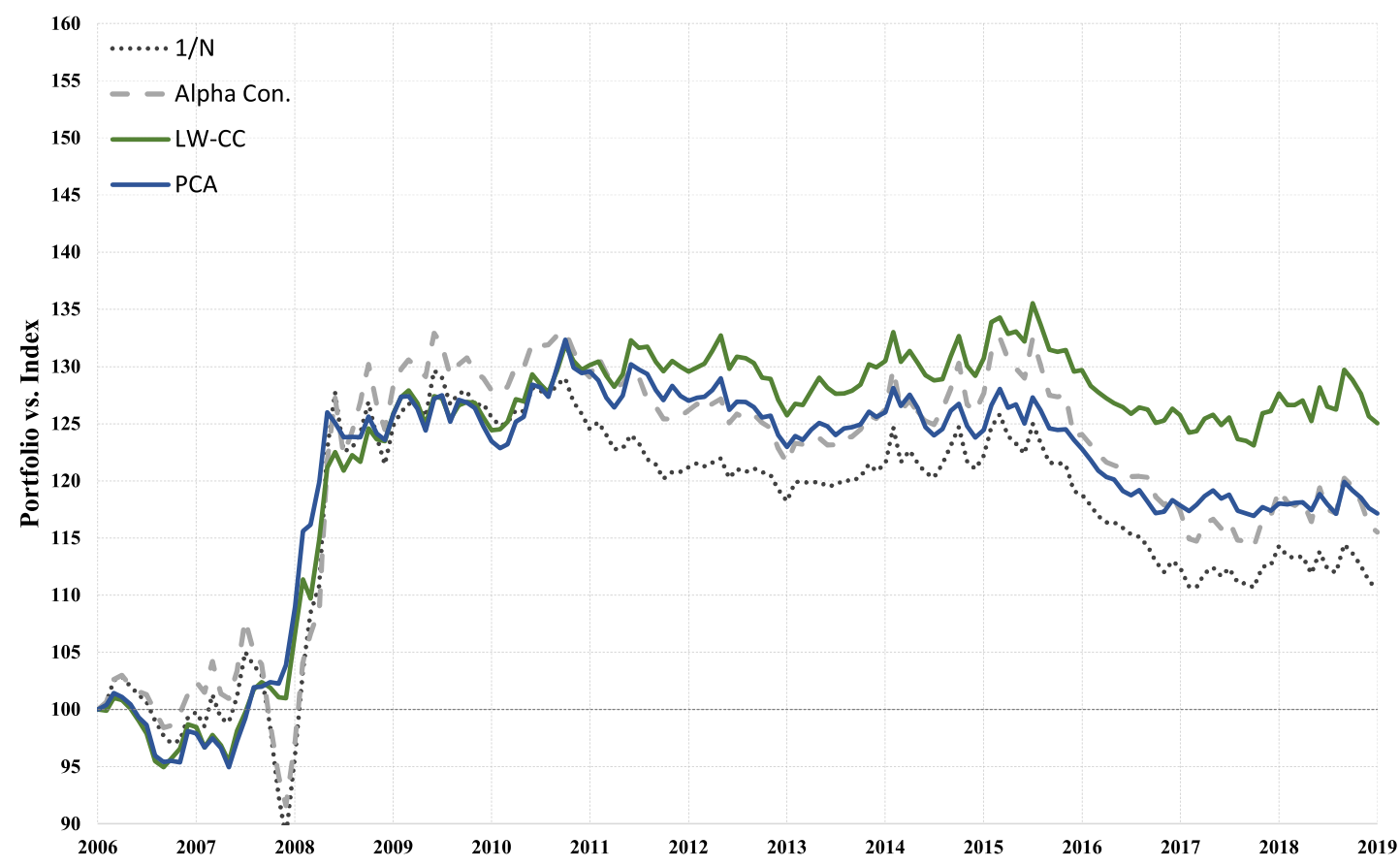

SPX Multifactor - LS 30\%/70\%

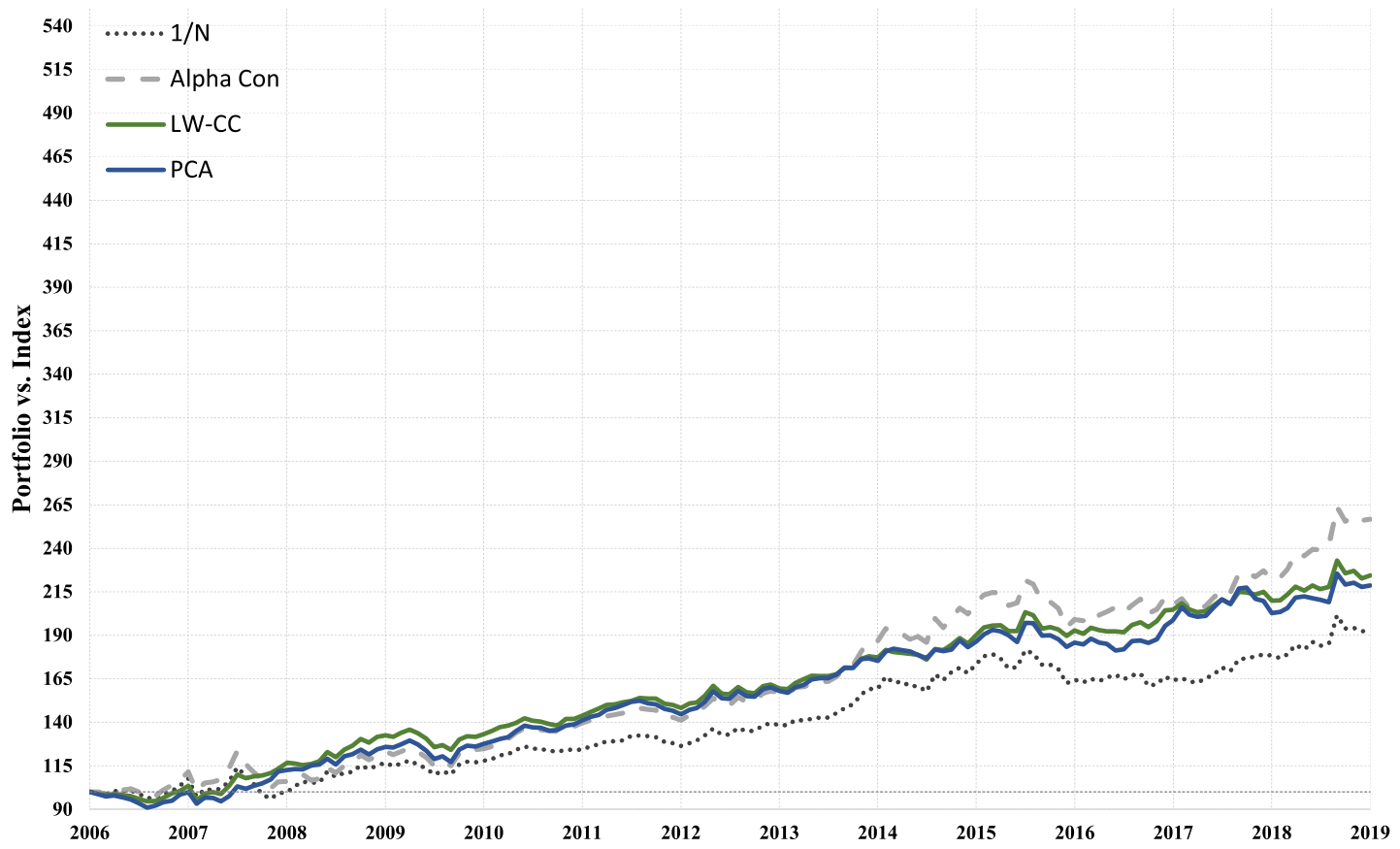

Fig. 7 Relative strength results of long-only $\left(q t^{+}=70 \%\right)$ and long-short $\left(q t^{-}=30 \%, q t^{+}=70 \%\right)$ SPX multifactor portfolios. The relative strength results of all approaches in relation to their underlying indices are displayed 
STX Multifactor - LO 70\%

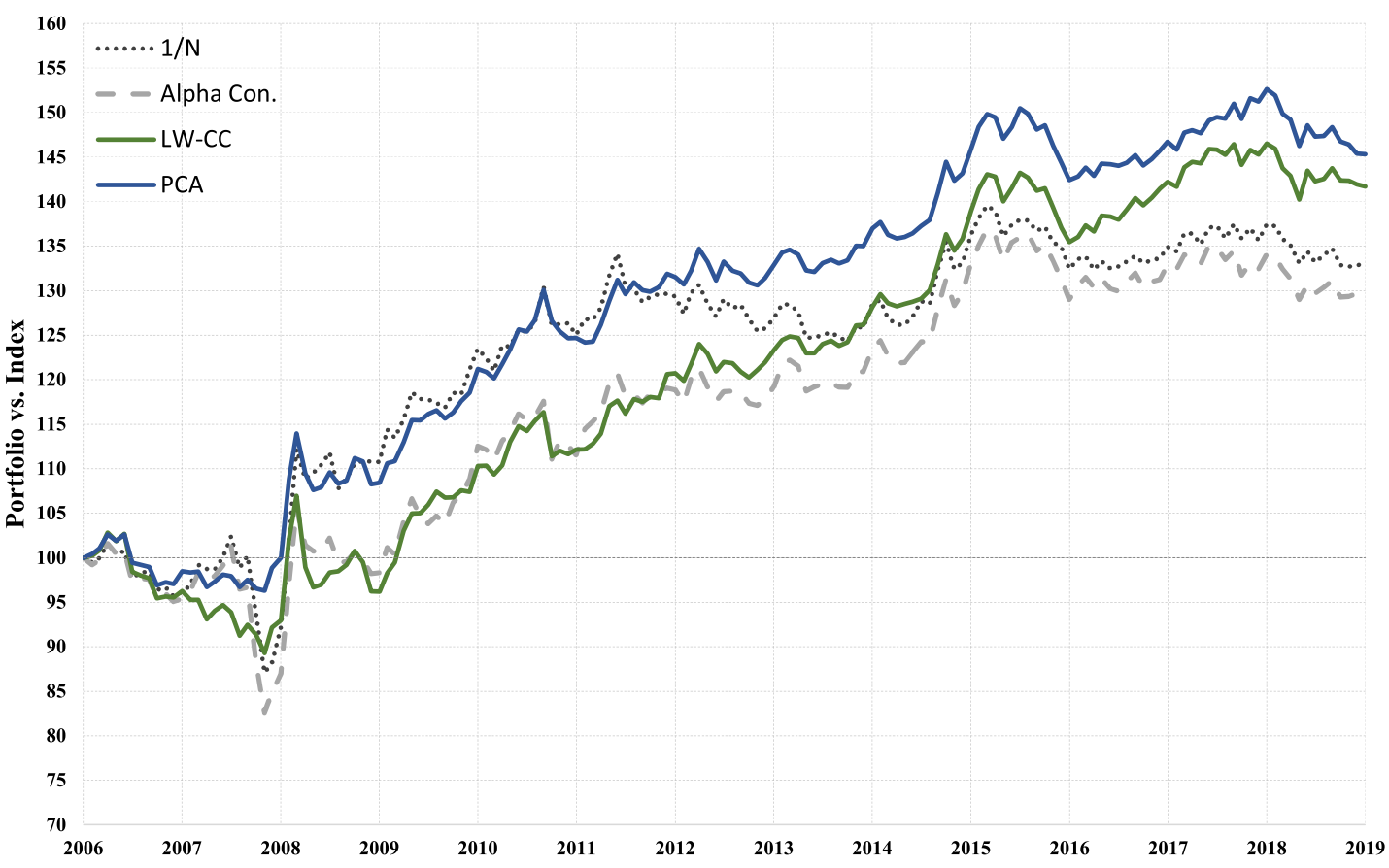

STX Multifactor - LS 30\%/70\%

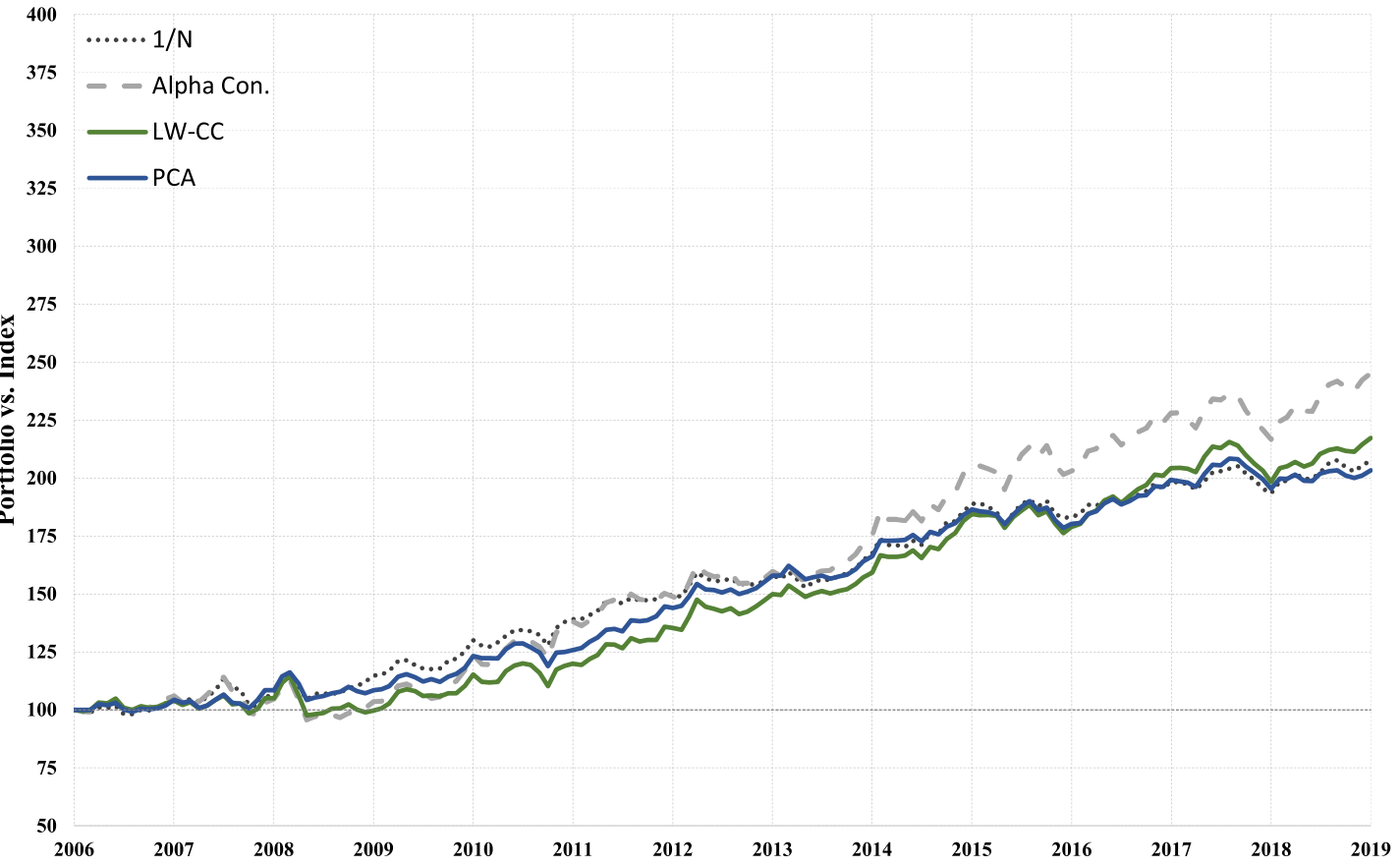

Fig. 8 Relative strength results of long-only $\left(q t^{+}=70 \%\right)$ and long-short $\left(q t^{-}=30 \%, q t^{+}=70 \%\right)$ STX multifactor portfolios. The relative strength results of all approaches in relation to their underlying indices are displayed 
Funding Open Access funding enabled and organized by Projekt DEAL.

Open Access This article is licensed under a Creative Commons Attribution 4.0 International License, which permits use, sharing, adaptation, distribution and reproduction in any medium or format, as long as you give appropriate credit to the original author(s) and the source, provide a link to the Creative Commons licence, and indicate if changes were made. The images or other third party material in this article are included in the article's Creative Commons licence, unless indicated otherwise in a credit line to the material. If material is not included in the article's Creative Commons licence and your intended use is not permitted by statutory regulation or exceeds the permitted use, you will need to obtain permission directly from the copyright holder. To view a copy of this licence, visit http://creativecommons.org/licenses/by/4.0/.

\section{References}

Amenc, N., F. Goltz, L. Martellini, and P. Retkowsky. 2011. Efficient indexation: An alternative to cap-weighted indices. Journal Of Investment Management (JOIM): Fourth Quarter.

Amenc, N., F. Goltz, and A. Lodh. 2012. Choose your betas: Benchmarking alternative equity index strategies. The Journal of Portfolio Management 39 (1): 88-111. https://doi.org/10.3905/jpm. 2012.39.1.088

Amenc, N., F. Ducolumbier, F. Goltz, and J. Ulhael, Ten Misconceptions about Smart Beta, 2016a.

Amenc, N., F. Ducoulombier, F. Goltz, A. Lodh, and S. Sivasubramanian. 2016b. Diversified or concentrated factor tilts? The Journal of Portfolio Management 42 (2): 64-76. https://doi.org/10.3905/ jpm.2016.42.2.064.

Amenc, N., F. Ducoulombier, M. Esakia, F. Goltz, and S. Sivasubramanian. 2017. Accounting for cross-factor interactions in multifactor portfolios without sacrificing diversification and risk control. The Journal of Portfolio Management 43 (5): 99-114. https://doi.org/ 10.3905/jpm.2017.43.5.099.

Arnott, R., C.R. Harvey, V. Kalesnik, and J. Linnainmaa. 2019. Alice's adventures in factorland: Three blunders that plague factor investing. The Journal of Portfolio Management 45 (4): 18-36. https:// doi.org/10.3905/jpm.2019.45.4.018.

Bai, J., and S. Ng. 2002. Determining the number of factors in approximate factor models. Econometrica 70 (1): 191-221. https://doi. org/10.1111/1468-0262.00273.

Bouchaud, J. P., and M. Potters. 2009. Financial applications of random matrix theory: a short review. arXiv preprint arXiv:0910.1205

Buckle, D. 2004. How to calculate breadth: An evolution of the fundamental law of active portfolio management. Journal of Asset Management 4 (6): 393-405. https://doi.org/10.1057/palgrave. jam.2240118.

Bun, J., J.-P. Bouchaud, and M. Potters. 2017. Cleaning large correlation matrices: tools from random matrix theory. Physics Reports 666: 1-109. https://doi.org/10.1016/j.physrep.2016.10.005.

Chow, T.-M., J.C. Hsu, L.-L. Kuo, and F. Li. 2014. A study of lowvolatility portfolio construction methods. The Journal of Portfolio Management 40 (4): 89-105. https://doi.org/10.3905/jpm.2014. 40.4.089.

Clarke, R., H. De Silva, and S. Thorley. 2002. Portfolio constraints and the fundamental law of active management. Financial Analysts Journal 58 (5): 48-66.

DeMiguel, V., A. Martín-Utrera, F.J. Nogales, and R. Uppal. 2020. A Transaction-Cost Perspective on the Multitude of Firm
Characteristics. The Review of Financial Studies 33 (5): 2180 2222. https://doi.org/10.1093/rfs/hhz085.

Ding, Z., D. Martin, and C. Yang. 2020. Portfolio Turnover when IC is Time Varying. Journal of Asset Management 21: 609-622. https:// doi.org/10.1057/s41260-019-00145-1.

Fan, J., Y. Fan, and J. Lv. 2008. High dimensional covariance matrix estimation using a factor model. Journal of Econometrics 147 (1): 186-197. https://doi.org/10.1016/j.jeconom.2008.09.017.

Fan, J., Y. Liao, and M. Mincheva. 2013. Large covariance estimation by thresholding principal orthogonal complements. Journal of the Royal Statistical Society: Series B (Statistical Methodology) 75 (4): 603-680. https://doi.org/10.1111/rssb.12016.

Fan, J., Y. Liao, and H. Liu. 2016. An overview of the estimation of large covariance and precision matrices. The Econometrics Journal 19 (1): C1-C32. https://doi.org/10.1111/ectj.12061.

Fan, J., Q. Sun, W.-X. Zhou, and Z. Zhu. 2018. Principal component analysis for big data. In Wiley StatsRef: Statistics Reference Online, pages 1-13. American Cancer Society, 2018. ISBN 9781118445112. https://doi.org/10.1002/9781118445112.stat0 8122.

Frazzini, A., R. Israel, and T. J. Moskowitz. 2018. Trading costs. Available at SSRN 3229719, 2018. https://doi.org/10.2139/ssrn.32297 19.

Ghayur, K., R. Heaney, and S. Platt. 2018. Constructing long-only multifactor strategies: Portfolio blending vs. signal blending. Financial Analysts Journal 74 (3): 70-85. https://doi.org/10.2469/faj. v74.n3.5.

Hallin, M., and R. Liška. 2007. Determining the number of factors in the general dynamic factor model. Journal of the American Statistical Association 102 (478): 603-617. https://doi.org/10.1198/ 016214506000001275 .

Heinrich, L., and M. Zurek. 2019. Alpha forecasting in factor investing: discriminating between the informational content of firm characteristics. Financial Markets and Portfolio Management 33 (3): 243-275. https://doi.org/10.1007/s11408-019-00333-4.

Hotelling, H. 1933. Analysis of a complex of statistical variables into principal components. Journal of Educational Psychology 24 (6): 417. https://doi.org/10.1037/h0071325.

Laloux, L., P. Cizeau, M. Potters, and J.-P. Bouchaud. 2000. Random matrix theory and financial correlations. International Journal of Theoretical and Applied Finance 03 (03): 391-397. https://doi. org/10.1142/S0219024900000255.

Ledoit, O., and M. Wolf. 2004. Honey, I shrunk the sample covariance matrix. The Journal of Portfolio Management 30 (4): 110-119. https://doi.org/10.3905/jpm.2004.110.

Ledoit, O., and M. Wolf. 2008. Robust performance hypothesis testing with the Sharpe ratio. Journal of Empirical Finance 15: 850-859. https://doi.org/10.1016/j.jempfin.2008.03.002.

Ledoit, O., and M. Wolf. 2015. Spectrum estimation: A unified framework for covariance matrix estimation and PCA in large dimensions. Journal of Multivariate Analysis 139: 360-384. https://doi. org/10.1016/j.jmva.2015.04.006.

MacKinlay, C.A., and L Pástor. 2000. Asset pricing models: Implications for expected returns and portfolio selection. The Review of Financial Studies 13 (4): 883-916. https://doi.org/10.1093/rfs/ 13.4.883.

Rothman, A.J., E. Levina, and J. Zhu. 2010. A new approach to Cholesky-based covariance regularization in high dimensions. Biometrika 97 (3): 539-550. https://doi.org/10.1093/biomet/ asq022.

Treynor, J.L., and F. Black. 1973. How to use security analysis to improve portfolio selection. The Journal of Business 46 (1): 66-86. 
Ye, J. 2008. How variation in signal quality affects performance. Financial Analysts Journal 64 (4): 48-61. https://doi.org/10.2469/faj. v64.n4.5.

Zurek, M., and L. Heinrich. 2021. Bottom-up versus top-down factor investing: an alpha forecasting perspective. Journal of Asset Management 22 (1): 11-29. https://doi.org/10.1057/ s41260-020-00188-9.

Publisher's Note Springer Nature remains neutral with regard to jurisdictional claims in published maps and institutional affiliations.

Lars Heinrich works as a portfolio manager in the department of liquid assets at W\&W Asset Management GmbH. He received an M.A. degree in applied science as well as a second M.A. degree in business administration with a focus on financial management at the Technical University of Freiberg. Apart from his activities as a portfolio manager, Lars Heinrich researches at the Europa Universität Viadrina in
Frankfurt (Oder). His research focus lies in factor investing and alpha forecasting.

Dr. Antoniya Shivarova works as a research assistant at the chair of Finance and Capital Market Theory of the Europa Universität Viadrina in Frankfurt (Oder), Germany, where she has recently received a Ph.D. degree in Business Administration with focus on finance. Apart from her activities as a research assistant, Dr. Antoniya Shivarova focuses in her research on covariance estimation, portfolio management, and machine learning applications in finance.

Martin Zurek works as a research assistant at the chair of finance and capital market theory of the Europa Universität Viadrina in Frankfurt (Oder). He received an M.Sc. and B.Sc. degree in business administration with a focus on finance at the Europa Universität Viadrina. Apart from his activities as a research assistant, Martin Zurek takes part in the Ph.D. program of the Europa Universität Viadrina. His research focuses on portfolio management and asset pricing. 